Supporting Information for:

\title{
Stimulation of P-glycoprotein ATPase by Analogues of Tetramethylrosamine. Coupling of Drug Binding at the " $R$ " Site to the ATP Hydrolysis Transition State
}

Gregory Tombline, ${ }^{*}$ David J. Donnelly, Michael K.Gannon, Jason J. Holt, Youngjae You, Mao Ye, Cara L. Nygren, and Michael R. Detty* 


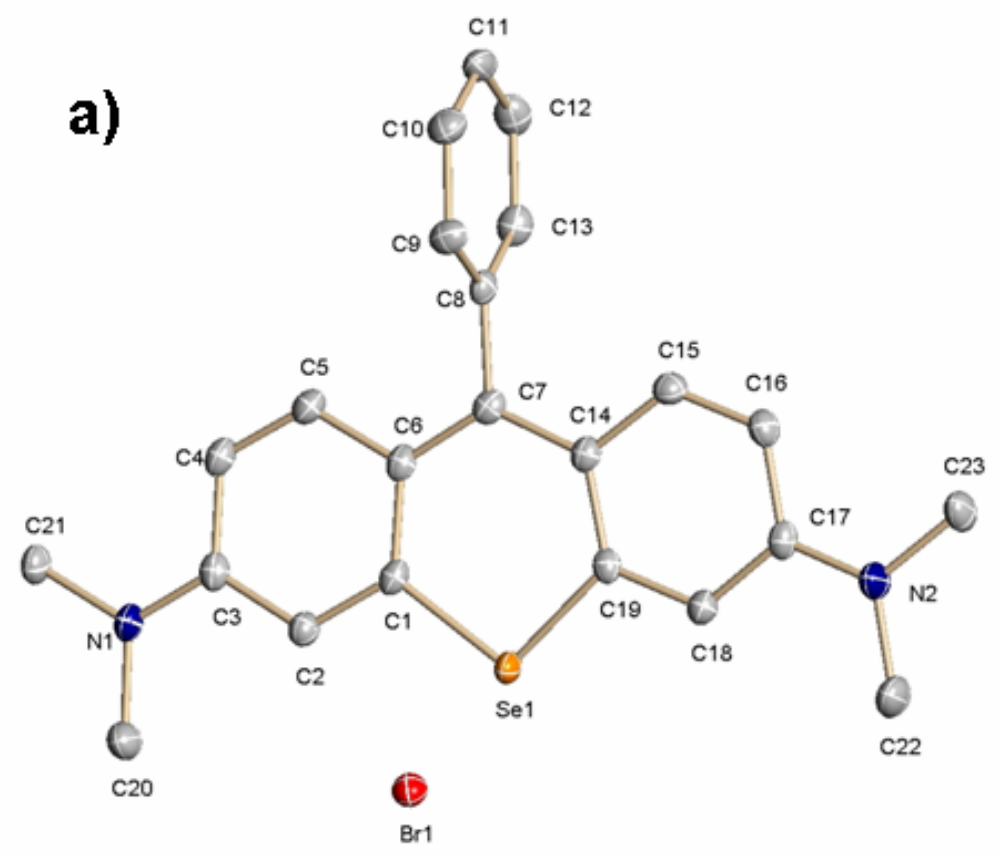

b)

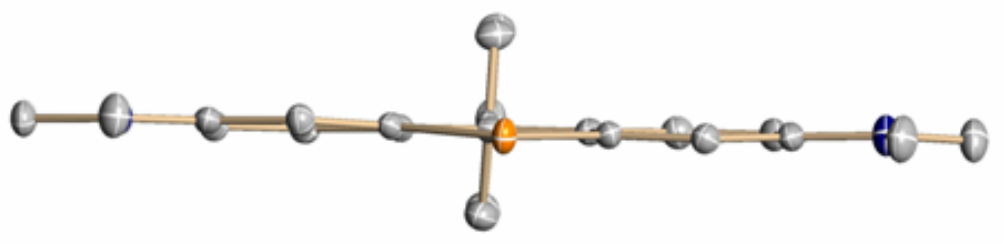

Fig.1S. ORTEP plot and label scheme for TMR-Se. Displacement ellipsoids are drawn at the $50 \%$ probability level viewed (a) from the top and (b) edge on down the Se1-C7 axis. Hydrogen atoms are omitted for clarity. 
Table 1S. Crystallographic Data for TMR-S and TMR-Se.

\begin{tabular}{|c|c|c|}
\hline & TMR-S & TMR-Se \\
\hline Formula & $\mathrm{C}_{23} \mathrm{H}_{23} \mathrm{~N}_{2} \mathrm{SBr}$ & $\mathrm{C}_{23} \mathrm{H}_{23} \mathrm{~N}_{2} \mathrm{SeBr}_{0.48} \mathrm{Cl}_{0.52}$ \\
\hline $\mathrm{M}_{\mathrm{r}}\left[\mathrm{g} \cdot \mathrm{mol}^{-1}\right]$ & 439.40 & 463.34 \\
\hline crystal shape/color & needle/dichroic & plate/dichroic \\
\hline crystal system & monoclinic & monoclinic \\
\hline space group & $\mathrm{P} 2{ }_{1} / \mathrm{c}$ & $\mathrm{P} 2{ }_{1} / \mathrm{c}$ \\
\hline$a[\AA]$ & $11.5873(9)$ & $9.2548(2)$ \\
\hline$b[\AA]$ & $18.277(2)$ & $28.7624(7)$ \\
\hline$c[\AA]$ & $10.3060(8)$ & $7.4136(2)$ \\
\hline$\alpha\left[^{\circ}\right]$ & 90 & 90 \\
\hline$\beta\left[^{\circ}\right]$ & $115.579(3)$ & $92.8050(10)$ \\
\hline$\gamma\left[{ }^{\circ}\right]$ & 90 & 90 \\
\hline $\mathrm{V}\left[\AA^{3}\right]$ & 1968.7(3) & $1971.06(8)$ \\
\hline$Z$ & 4 & 4 \\
\hline$\rho_{\text {calc }}\left[\mathrm{g} / \mathrm{cm}^{3}\right]$ & 1.483 & 1.561 \\
\hline$\mu\left[\mathrm{mm}^{-1}\right]$ & 2.205 & 2.96 \\
\hline $\mathrm{T}[\mathrm{K}]$ & $90(1)$ & $90(1)$ \\
\hline$\lambda(\AA)$ & 0.71073 & 0.71073 \\
\hline $\operatorname{Max} .2 \theta\left[^{\circ}\right]$ & 58.4 & 52.3 \\
\hline abs corr method & SADABS 2.10 & SADABS 2.10 \\
\hline no. of reflns measd & 32448 & 27119 \\
\hline no. of unique reflns $\left(\mathrm{R}_{\mathrm{int}}\right)$ & $5322[0.0244]$ & $4071[0.0274]$ \\
\hline no. params refined & 248 & 249 \\
\hline$R[I>2 \sigma(I)]$ & 0.0286 & 0.0226 \\
\hline$w R_{2}[I>2 \sigma(I)]$ & 0.0770 & 0.0578 \\
\hline GOF & 1.028 & 1.042 \\
\hline
\end{tabular}


Table 2S. Atomic coordinates $\left(10^{4}\right)$ and equivalent isotropic displacement parameters $\left(\AA^{2} \times 10^{3}\right)$ for TMR-S.

\begin{tabular}{|c|c|c|c|c|}
\hline & $\mathrm{x}$ & $\mathrm{y}$ & z & $\mathrm{U}(\mathrm{eq})$ \\
\hline $\operatorname{Br}(1)$ & $4319(1)$ & $3548(1)$ & $2937(1)$ & $20(1)$ \\
\hline $\mathrm{C}(1)$ & $2039(2)$ & $4972(1)$ & $9605(2)$ & $13(1)$ \\
\hline$C(2)$ & $3303(2)$ & $5087(1)$ & $10552(2)$ & $15(1)$ \\
\hline$C(3)$ & $3986(2)$ & $5691(1)$ & $10393(2)$ & $15(1)$ \\
\hline C(4) & $3304(2)$ & $6197(1)$ & $9253(2)$ & $17(1)$ \\
\hline$C(5)$ & $2048(2)$ & $6086(1)$ & $8357(2)$ & $16(1)$ \\
\hline$C(6)$ & $1344(2)$ & $5464(1)$ & $8445(2)$ & $14(1)$ \\
\hline$C(7)$ & $50(2)$ & $5360(1)$ & $7464(2)$ & $13(1)$ \\
\hline $\mathrm{C}(8)$ & $-555(2)$ & $5931(1)$ & $6325(2)$ & $14(1)$ \\
\hline C(9) & $-177(2)$ & $6016(1)$ & $5214(2)$ & $16(1)$ \\
\hline$C(10)$ & $-702(2)$ & $6575(1)$ & $4209(2)$ & $19(1)$ \\
\hline $\mathrm{C}(11)$ & $-1599(2)$ & $7050(1)$ & $4298(2)$ & 21(1) \\
\hline$C(12)$ & $-1998(2)$ & $6957(1)$ & $5381(2)$ & $22(1)$ \\
\hline$C(13)$ & $-1485(2)$ & $6398(1)$ & $6386(2)$ & $19(1)$ \\
\hline$C(14)$ & $-698(2)$ & $4762(1)$ & $7516(2)$ & $14(1)$ \\
\hline$C(15)$ & $-1995(2)$ & $4668(1)$ & $6480(2)$ & $17(1)$ \\
\hline$C(16)$ & $-2736(2)$ & $4098(1)$ & $6510(2)$ & $18(1)$ \\
\hline$C(17)$ & $-2248(2)$ & $3562(1)$ & $7631(2)$ & $16(1)$ \\
\hline $\mathrm{C}(18)$ & $-962(2)$ & $3628(1)$ & $8651(2)$ & $16(1)$ \\
\hline C(19) & $-211(2)$ & $4205(1)$ & $8592(2)$ & $14(1)$ \\
\hline$C(20)$ & $5915(2)$ & $5341(1)$ & $12530(2)$ & $20(1)$ \\
\hline $\mathrm{C}(21)$ & $5941(2)$ & $6409(1)$ & $11018(2)$ & $18(1)$ \\
\hline $\mathrm{C}(22)$ & $-2507(2)$ & $2473(1)$ & $8855(2)$ & $19(1)$ \\
\hline$C(23)$ & $-4356(2)$ & $2984(1)$ & $6680(2)$ & $20(1)$ \\
\hline $\mathrm{N}(1)$ & $5230(1)$ & $5804(1)$ & $11272(2)$ & $16(1)$ \\
\hline $\mathrm{N}(2)$ & $-2996(2)$ & $3011(1)$ & $7695(2)$ & $18(1)$ \\
\hline $\mathrm{S}(1)$ & $1352(1)$ & $4186(1)$ & $9902(1)$ & $15(1)$ \\
\hline
\end{tabular}


Table 3S. Anisotropic displacement parameters $\left(\AA^{2} \times 10^{3}\right)$ for TMR-S.

\begin{tabular}{|c|c|c|c|c|c|c|}
\hline & $\mathrm{U}^{11}$ & $\mathrm{U}^{22}$ & $\mathrm{U}^{33}$ & $\mathrm{U}^{23}$ & $\mathrm{U}^{13}$ & $\mathrm{U}^{12}$ \\
\hline $\operatorname{Br}(1)$ & $18(1)$ & $21(1)$ & $20(1)$ & $5(1)$ & $6(1)$ & $3(1)$ \\
\hline$C(1)$ & $15(1)$ & $13(1)$ & $13(1)$ & $0(1)$ & $7(1)$ & $1(1)$ \\
\hline$C(2)$ & $15(1)$ & $15(1)$ & $12(1)$ & 1(1) & $4(1)$ & $2(1)$ \\
\hline$C(3)$ & $16(1)$ & $15(1)$ & $12(1)$ & $-2(1)$ & $5(1)$ & 1(1) \\
\hline$C(4)$ & $18(1)$ & $15(1)$ & $16(1)$ & $1(1)$ & $5(1)$ & $-1(1)$ \\
\hline$C(5)$ & $19(1)$ & $14(1)$ & $14(1)$ & $2(1)$ & $5(1)$ & 1(1) \\
\hline$C(6)$ & $16(1)$ & $14(1)$ & $12(1)$ & $0(1)$ & $6(1)$ & $1(1)$ \\
\hline$C(7)$ & $14(1)$ & $14(1)$ & $12(1)$ & 1(1) & $6(1)$ & $2(1)$ \\
\hline $\mathrm{C}(8)$ & $14(1)$ & $15(1)$ & $12(1)$ & 1(1) & $4(1)$ & $0(1)$ \\
\hline $\mathrm{C}(9)$ & $16(1)$ & $17(1)$ & $14(1)$ & $-1(1)$ & $6(1)$ & $-1(1)$ \\
\hline$C(10)$ & $23(1)$ & $21(1)$ & $12(1)$ & 1(1) & $6(1)$ & $-5(1)$ \\
\hline $\mathrm{C}(11)$ & $23(1)$ & $16(1)$ & $15(1)$ & $3(1)$ & 1(1) & $-2(1)$ \\
\hline$C(12)$ & $21(1)$ & $20(1)$ & $21(1)$ & $2(1)$ & $6(1)$ & $5(1)$ \\
\hline$C(13)$ & $20(1)$ & $21(1)$ & $17(1)$ & $3(1)$ & $9(1)$ & $5(1)$ \\
\hline$C(14)$ & $15(1)$ & $16(1)$ & $11(1)$ & $1(1)$ & $6(1)$ & $1(1)$ \\
\hline$C(15)$ & $15(1)$ & $20(1)$ & $13(1)$ & $3(1)$ & $4(1)$ & 1(1) \\
\hline$C(16)$ & $14(1)$ & $21(1)$ & $16(1)$ & $1(1)$ & $5(1)$ & $0(1)$ \\
\hline$C(17)$ & $16(1)$ & $17(1)$ & $16(1)$ & $-1(1)$ & $8(1)$ & $-1(1)$ \\
\hline $\mathrm{C}(18)$ & $17(1)$ & $16(1)$ & $15(1)$ & $2(1)$ & $7(1)$ & $1(1)$ \\
\hline$C(19)$ & $15(1)$ & $15(1)$ & $12(1)$ & $0(1)$ & $6(1)$ & 1(1) \\
\hline $\mathrm{C}(20)$ & $18(1)$ & $20(1)$ & $17(1)$ & $3(1)$ & $2(1)$ & $2(1)$ \\
\hline $\mathrm{C}(21)$ & $17(1)$ & $17(1)$ & $19(1)$ & $0(1)$ & $6(1)$ & $-3(1)$ \\
\hline$C(22)$ & $19(1)$ & $16(1)$ & $20(1)$ & $1(1)$ & $8(1)$ & $-1(1)$ \\
\hline $\mathrm{C}(23)$ & $17(1)$ & $19(1)$ & $21(1)$ & $0(1)$ & $6(1)$ & $-2(1)$ \\
\hline $\mathrm{N}(1)$ & $15(1)$ & $15(1)$ & $14(1)$ & $1(1)$ & $3(1)$ & $-1(1)$ \\
\hline $\mathrm{N}(2)$ & $17(1)$ & $18(1)$ & $18(1)$ & 2(1) & $7(1)$ & $-2(1)$ \\
\hline$S(1)$ & $14(1)$ & $15(1)$ & $13(1)$ & $3(1)$ & $4(1)$ & $0(1)$ \\
\hline
\end{tabular}


Table 4S. Bond lengths $[\AA]$ and angles $\left[^{\circ}\right]$ for TMR-S.

\begin{tabular}{|c|c|}
\hline $\mathrm{C}(1)-\mathrm{C}(2)$ & $1.381(2)$ \\
\hline$C(1)-C(6)$ & $1.433(2)$ \\
\hline $\mathrm{C}(1)-\mathrm{S}(1)$ & $1.7337(17)$ \\
\hline $\mathrm{C}(2)-\mathrm{C}(3)$ & $1.409(2)$ \\
\hline $\mathrm{C}(2)-\mathrm{H}(2)$ & 0.9500 \\
\hline $\mathrm{C}(3)-\mathrm{N}(1)$ & $1.347(2)$ \\
\hline $\mathrm{C}(3)-\mathrm{C}(4)$ & $1.434(2)$ \\
\hline $\mathrm{C}(4)-\mathrm{C}(5)$ & $1.361(2)$ \\
\hline $\mathrm{C}(4)-\mathrm{H}(4)$ & 0.9500 \\
\hline$C(5)-C(6)$ & $1.425(2)$ \\
\hline $\mathrm{C}(5)-\mathrm{H}(5)$ & 0.9500 \\
\hline $\mathrm{C}(6)-\mathrm{C}(7)$ & $1.413(2)$ \\
\hline$C(7)-C(14)$ & $1.411(2)$ \\
\hline$C(7)-C(8)$ & $1.499(2)$ \\
\hline $\mathrm{C}(8)-\mathrm{C}(13)$ & $1.397(2)$ \\
\hline $\mathrm{C}(8)-\mathrm{C}(9)$ & $1.400(2)$ \\
\hline C(9)-C(10) & $1.394(2)$ \\
\hline C(9)-H(9) & 0.9500 \\
\hline $\mathrm{C}(10)-\mathrm{C}(11)$ & $1.388(3)$ \\
\hline $\mathrm{C}(10)-\mathrm{H}(10)$ & 0.9500 \\
\hline C(11)-C(12) & $1.391(3)$ \\
\hline C(11)-H(11) & 0.9500 \\
\hline C(12)-C(13) & $1.392(3)$ \\
\hline $\mathrm{C}(12)-\mathrm{H}(12)$ & 0.9500 \\
\hline C(13)-H(13) & 0.9500 \\
\hline $\mathrm{C}(14)-\mathrm{C}(15)$ & $1.429(2)$ \\
\hline C(14)-C(19) & $1.430(2)$ \\
\hline$C(15)-C(16)$ & $1.358(3)$ \\
\hline C(15)-H(15) & 0.9500 \\
\hline$C(16)-C(17)$ & $1.432(2)$ \\
\hline $\mathrm{C}(16)-\mathrm{H}(16)$ & 0.9500 \\
\hline $\mathrm{C}(17)-\mathrm{N}(2)$ & $1.347(2)$ \\
\hline C(17)-C(18) & $1.410(2)$ \\
\hline C(18)-C(19) & $1.384(2)$ \\
\hline C(18)-H(18) & 0.9500 \\
\hline C(19)-S(1) & $1.7303(17)$ \\
\hline $\mathrm{C}(20)-\mathrm{N}(1)$ & $1.463(2)$ \\
\hline $\mathrm{C}(20)-\mathrm{H}(20 \mathrm{~A})$ & 0.9800 \\
\hline $\mathrm{C}(20)-\mathrm{H}(20 \mathrm{~B})$ & 0.9800 \\
\hline $\mathrm{C}(20)-\mathrm{H}(20 \mathrm{C})$ & 0.9800 \\
\hline $\mathrm{C}(21)-\mathrm{N}(1)$ & $1.468(2)$ \\
\hline $\mathrm{C}(21)-\mathrm{H}(21 \mathrm{~A})$ & 0.9800 \\
\hline $\mathrm{C}(21)-\mathrm{H}(21 \mathrm{~B})$ & 0.9800 \\
\hline C(21)-H(21C) & 0.9800 \\
\hline $\mathrm{C}(22)-\mathrm{N}(2)$ & $1.460(2)$ \\
\hline $\mathrm{C}(22)-\mathrm{H}(22 \mathrm{~A})$ & 0.9800 \\
\hline $\mathrm{C}(22)-\mathrm{H}(22 \mathrm{~B})$ & 0.9800 \\
\hline $\mathrm{C}(22)-\mathrm{H}(22 \mathrm{C})$ & 0.9800 \\
\hline $\mathrm{C}(23)-\mathrm{N}(2)$ & $1.469(2)$ \\
\hline $\mathrm{C}(23)-\mathrm{H}(23 \mathrm{~A})$ & 0.9800 \\
\hline $\mathrm{C}(23)-\mathrm{H}(23 \mathrm{~B})$ & 0.9800 \\
\hline $\mathrm{C}(23)-\mathrm{H}(23 \mathrm{C})$ & 0.9800 \\
\hline
\end{tabular}




\begin{tabular}{|c|c|}
\hline$C(2)-C(1)-C(6)$ & $122.50(16)$ \\
\hline $\mathrm{C}(2)-\mathrm{C}(1)-\mathrm{S}(1)$ & $115.40(13)$ \\
\hline $\mathrm{C}(6)-\mathrm{C}(1)-\mathrm{S}(1)$ & $122.09(13)$ \\
\hline $\mathrm{C}(1)-\mathrm{C}(2)-\mathrm{C}(3)$ & $120.94(15)$ \\
\hline $\mathrm{C}(1)-\mathrm{C}(2)-\mathrm{H}(2)$ & 119.5 \\
\hline $\mathrm{C}(3)-\mathrm{C}(2)-\mathrm{H}(2)$ & 119.5 \\
\hline $\mathrm{N}(1)-\mathrm{C}(3)-\mathrm{C}(2)$ & $122.21(16)$ \\
\hline $\mathrm{N}(1)-\mathrm{C}(3)-\mathrm{C}(4)$ & $120.29(16)$ \\
\hline$C(2)-C(3)-C(4)$ & $117.50(15)$ \\
\hline$C(5)-C(4)-C(3)$ & $120.66(16)$ \\
\hline $\mathrm{C}(5)-\mathrm{C}(4)-\mathrm{H}(4)$ & 119.7 \\
\hline $\mathrm{C}(3)-\mathrm{C}(4)-\mathrm{H}(4)$ & 119.7 \\
\hline$C(4)-C(5)-C(6)$ & $123.34(16)$ \\
\hline $\mathrm{C}(4)-\mathrm{C}(5)-\mathrm{H}(5)$ & 118.3 \\
\hline $\mathrm{C}(6)-\mathrm{C}(5)-\mathrm{H}(5)$ & 118.3 \\
\hline$C(7)-C(6)-C(5)$ & $121.38(15)$ \\
\hline$C(7)-C(6)-C(1)$ & $123.66(15)$ \\
\hline$C(5)-C(6)-C(1)$ & $114.95(15)$ \\
\hline$C(14)-C(7)-C(6)$ & $123.75(15)$ \\
\hline $\mathrm{C}(14)-\mathrm{C}(7)-\mathrm{C}(8)$ & $118.85(15)$ \\
\hline C(6)-C(7)-C(8) & $117.39(15)$ \\
\hline $\mathrm{C}(13)-\mathrm{C}(8)-\mathrm{C}(9)$ & $119.27(16)$ \\
\hline $\mathrm{C}(13)-\mathrm{C}(8)-\mathrm{C}(7)$ & $119.89(15)$ \\
\hline C(9)-C(8)-C(7) & $120.82(15)$ \\
\hline $\mathrm{C}(10)-\mathrm{C}(9)-\mathrm{C}(8)$ & $119.94(17)$ \\
\hline $\mathrm{C}(10)-\mathrm{C}(9)-\mathrm{H}(9)$ & 120.0 \\
\hline $\mathrm{C}(8)-\mathrm{C}(9)-\mathrm{H}(9)$ & 120.0 \\
\hline $\mathrm{C}(11)-\mathrm{C}(10)-\mathrm{C}(9)$ & $120.59(17)$ \\
\hline $\mathrm{C}(11)-\mathrm{C}(10)-\mathrm{H}(10)$ & 119.7 \\
\hline $\mathrm{C}(9)-\mathrm{C}(10)-\mathrm{H}(10)$ & 119.7 \\
\hline $\mathrm{C}(10)-\mathrm{C}(11)-\mathrm{C}(12)$ & $119.58(17)$ \\
\hline $\mathrm{C}(10)-\mathrm{C}(11)-\mathrm{H}(11)$ & 120.2 \\
\hline $\mathrm{C}(12)-\mathrm{C}(11)-\mathrm{H}(11)$ & 120.2 \\
\hline $\mathrm{C}(11)-\mathrm{C}(12)-\mathrm{C}(13)$ & $120.27(18)$ \\
\hline $\mathrm{C}(11)-\mathrm{C}(12)-\mathrm{H}(12)$ & 119.9 \\
\hline $\mathrm{C}(13)-\mathrm{C}(12)-\mathrm{H}(12)$ & 119.9 \\
\hline $\mathrm{C}(12)-\mathrm{C}(13)-\mathrm{C}(8)$ & $120.30(17)$ \\
\hline $\mathrm{C}(12)-\mathrm{C}(13)-\mathrm{H}(13)$ & 119.8 \\
\hline $\mathrm{C}(8)-\mathrm{C}(13)-\mathrm{H}(13)$ & 119.8 \\
\hline C(7)-C(14)-C(15) & $121.99(15)$ \\
\hline C(7)-C(14)-C(19) & $122.59(15)$ \\
\hline$C(15)-C(14)-C(19)$ & $115.41(15)$ \\
\hline$C(16)-C(15)-C(14)$ & $123.10(16)$ \\
\hline $\mathrm{C}(16)-\mathrm{C}(15)-\mathrm{H}(15)$ & 118.4 \\
\hline $\mathrm{C}(14)-\mathrm{C}(15)-\mathrm{H}(15)$ & 118.4 \\
\hline$C(15)-C(16)-C(17)$ & $120.69(16)$ \\
\hline $\mathrm{C}(15)-\mathrm{C}(16)-\mathrm{H}(16)$ & 119.7 \\
\hline $\mathrm{C}(17)-\mathrm{C}(16)-\mathrm{H}(16)$ & 119.7 \\
\hline $\mathrm{N}(2)-\mathrm{C}(17)-\mathrm{C}(18)$ & $121.55(16)$ \\
\hline $\mathrm{N}(2)-\mathrm{C}(17)-\mathrm{C}(16)$ & $120.82(16)$ \\
\hline $\mathrm{C}(18)-\mathrm{C}(17)-\mathrm{C}(16)$ & $117.63(16)$ \\
\hline $\mathrm{C}(19)-\mathrm{C}(18)-\mathrm{C}(17)$ & $120.98(16)$ \\
\hline C(19)-C(18)-H(18) & 119.5 \\
\hline $\mathrm{C}(17)-\mathrm{C}(18)-\mathrm{H}(18)$ & 119.5 \\
\hline$C(18)-C(19)-C(14)$ & $122.11(16)$ \\
\hline
\end{tabular}




$\begin{array}{ll}\mathrm{C}(18)-\mathrm{C}(19)-\mathrm{S}(1) & 114.57(13) \\ \mathrm{C}(14)-\mathrm{C}(19)-\mathrm{S}(1) & 123.32(13) \\ \mathrm{N}(1)-\mathrm{C}(20)-\mathrm{H}(20 \mathrm{~A}) & 109.5 \\ \mathrm{~N}(1)-\mathrm{C}(20)-\mathrm{H}(20 \mathrm{~B}) & 109.5 \\ \mathrm{H}(20 \mathrm{~A})-\mathrm{C}(20)-\mathrm{H}(20 \mathrm{~B}) & 109.5 \\ \mathrm{~N}(1)-\mathrm{C}(20)-\mathrm{H}(20 \mathrm{C}) & 109.5 \\ \mathrm{H}(20 \mathrm{~A})-\mathrm{C}(20)-\mathrm{H}(20 \mathrm{C}) & 109.5 \\ \mathrm{H}(20 \mathrm{~B})-\mathrm{C}(20)-\mathrm{H}(20 \mathrm{C}) & 109.5 \\ \mathrm{~N}(1)-\mathrm{C}(21)-\mathrm{H}(21 \mathrm{~A}) & 109.5 \\ \mathrm{~N}(1)-\mathrm{C}(21)-\mathrm{H}(21 \mathrm{~B}) & 109.5 \\ \mathrm{H}(21 \mathrm{~A})-\mathrm{C}(21)-\mathrm{H}(21 \mathrm{~B}) & 109.5 \\ \mathrm{~N}(1)-\mathrm{C}(21)-\mathrm{H}(21 \mathrm{C}) & 109.5 \\ \mathrm{H}(21 \mathrm{~A})-\mathrm{C}(21)-\mathrm{H}(21 \mathrm{C}) & 109.5 \\ \mathrm{H}(21 \mathrm{~B})-\mathrm{C}(21)-\mathrm{H}(21 \mathrm{C}) & 109.5 \\ \mathrm{~N}(2)-\mathrm{C}(22)-\mathrm{H}(22 \mathrm{~A}) & 109.5 \\ \mathrm{~N}(2)-\mathrm{C}(22)-\mathrm{H}(22 \mathrm{~B}) & 109.5 \\ \mathrm{H}(22 \mathrm{~A})-\mathrm{C}(22)-\mathrm{H}(22 \mathrm{~B}) & 109.5 \\ \mathrm{~N}(2)-\mathrm{C}(22)-\mathrm{H}(22 \mathrm{C}) & 109.5 \\ \mathrm{H}(22 \mathrm{~A})-\mathrm{C}(22)-\mathrm{H}(22 \mathrm{C}) & 109.5 \\ \mathrm{H}(22 \mathrm{~B})-\mathrm{C}(22)-\mathrm{H}(22 \mathrm{C}) & 109.5 \\ \mathrm{~N}(2)-\mathrm{C}(23)-\mathrm{H}(23 \mathrm{~A}) & 109.5 \\ \mathrm{~N}(2)-\mathrm{C}(23)-\mathrm{H}(23 \mathrm{~B}) & 109.5 \\ \mathrm{H}(23 \mathrm{~A})-\mathrm{C}(23)-\mathrm{H}(23 \mathrm{~B}) & 109.5 \\ \text { N(2)-C(23)-H(23C) } & 109.5 \\ \mathrm{H}(23 \mathrm{~A})-\mathrm{C}(23)-\mathrm{H}(23 \mathrm{C}) & 109.5 \\ \mathrm{H}(23 \mathrm{~B})-\mathrm{C}(23)-\mathrm{H}(23 \mathrm{C}) & 109.5 \\ \mathrm{C}(3)-\mathrm{N}(1)-\mathrm{C}(20) & 121.46(15) \\ \mathrm{C}(3)-\mathrm{N}(1)-\mathrm{C}(21) & 121.01(15) \\ \mathrm{C}(20)-\mathrm{N}(1)-\mathrm{C}(21) & 117.52(15) \\ \mathrm{C}(17)-\mathrm{N}(2)-\mathrm{C}(22) & 120.79(15) \\ \mathrm{C}(17)-\mathrm{N}(2)-\mathrm{C}(23) & 120.39(15) \\ \mathrm{C}(22)-\mathrm{N}(2)-\mathrm{C}(23) & 118.55(15) \\ \mathrm{C}(19)-\mathrm{S}(1)-\mathrm{C}(1) & 104.55(8) \\ & \end{array}$


Table 5S. Atomic coordinates $\left(10^{4}\right)$ and equivalent isotropic displacement parameters $\left(\AA^{2} \times 10^{3}\right)$ for TMR-Se.

\begin{tabular}{|c|c|c|c|c|}
\hline & $\mathrm{x}$ & $\mathrm{y}$ & $\mathrm{z}$ & $\mathrm{U}(\mathrm{eq})$ \\
\hline $\operatorname{Br}(1)$ & $3149(1)$ & $4486(1)$ & $2026(1)$ & $20(1)$ \\
\hline $\mathrm{C}(18)$ & $3835(2)$ & $6297(1)$ & $-2270(2)$ & $18(1)$ \\
\hline$C(19)$ & $3009(2)$ & $6222(1)$ & $-787(2)$ & $16(1)$ \\
\hline $\mathrm{C}(17)$ & $3432(2)$ & $6631(1)$ & $-3584(2)$ & $19(1)$ \\
\hline $\mathrm{C}(8)$ & $-551(2)$ & $6694(1)$ & $958(2)$ & $18(1)$ \\
\hline$C(23)$ & $3810(2)$ & $7042(1)$ & $-6412(3)$ & $26(1)$ \\
\hline$C(15)$ & $1304(2)$ & $6794(1)$ & $-1916(2)$ & $20(1)$ \\
\hline$C(14)$ & $1697(2)$ & $6467(1)$ & $-537(2)$ & $17(1)$ \\
\hline$C(7)$ & $788(2)$ & $6400(1)$ & $954(2)$ & $17(1)$ \\
\hline$C(11)$ & $-2995(2)$ & $7262(1)$ & $852(3)$ & $24(1)$ \\
\hline$C(10)$ & $-1705(2)$ & $7422(1)$ & 1641(3) & $25(1)$ \\
\hline$C(16)$ & $2115(2)$ & $6876(1)$ & $-3370(2)$ & $20(1)$ \\
\hline$C(12)$ & $-3071(2)$ & $6818(1)$ & 142(3) & $26(1)$ \\
\hline $\mathrm{C}(9)$ & $-491(2)$ & $7139(1)$ & $1698(3)$ & $22(1)$ \\
\hline$C(13)$ & $-1859(2)$ & $6532(1)$ & $185(3)$ & $23(1)$ \\
\hline $\mathrm{C}(22)$ & $5621(2)$ & $6457(1)$ & $-5201(3)$ & $30(1)$ \\
\hline $\mathrm{C}(2)$ & $2593(2)$ & $5523(1)$ & $4067(2)$ & $19(1)$ \\
\hline$C(4)$ & 273(2) & $5755(1)$ & $5231(2)$ & $20(1)$ \\
\hline $\mathrm{C}(1)$ & $2334(2)$ & $5805(1)$ & $2597(2)$ & $17(1)$ \\
\hline$C(20)$ & $3143(2)$ & 4907(1) & $6998(3)$ & $27(1)$ \\
\hline $\mathrm{C}(5)$ & $56(2)$ & $6045(1)$ & $3800(2)$ & $19(1)$ \\
\hline$C(3)$ & $1576(2)$ & $5483(1)$ & $5430(2)$ & $18(1)$ \\
\hline$C(6)$ & $1065(2)$ & $6093(1)$ & $2385(2)$ & $17(1)$ \\
\hline $\mathrm{C}(21)$ & $779(2)$ & $5151(1)$ & $8243(2)$ & $22(1)$ \\
\hline $\mathrm{Cl}(1)$ & $3149(1)$ & $4486(1)$ & 2026(1) & $20(1)$ \\
\hline $\mathrm{N}(2)$ & $4278(2)$ & $6719(1)$ & $-4992(2)$ & $23(1)$ \\
\hline $\mathrm{N}(1)$ & $1818(2)$ & $5196(1)$ & $6830(2)$ & $20(1)$ \\
\hline $\operatorname{Se}(1)$ & $3732(1)$ & $5770(1)$ & $846(1)$ & $18(1)$ \\
\hline
\end{tabular}


Table 6S. Anisotropic displacement parameters $\left(\AA^{2} \times 10^{3}\right)$ for TMR-Se.

\begin{tabular}{lcccccc}
\hline & $\mathrm{U}^{11}$ & $\mathrm{U}^{22}$ & $\mathrm{U}^{33}$ & $\mathrm{U}^{23}$ & $\mathrm{U}^{13}$ & $\mathrm{U}^{12}$ \\
\hline $\mathrm{Br}(1)$ & $22(1)$ & $17(1)$ & $21(1)$ & $-1(1)$ & $2(1)$ & $2(1)$ \\
$\mathrm{C}(18)$ & $20(1)$ & $18(1)$ & $16(1)$ & $-1(1)$ & $3(1)$ & $-1(1)$ \\
$\mathrm{C}(19)$ & $21(1)$ & $14(1)$ & $14(1)$ & $0(1)$ & $1(1)$ & $-2(1)$ \\
$\mathrm{C}(17)$ & $22(1)$ & $20(1)$ & $15(1)$ & $0(1)$ & $3(1)$ & $-6(1)$ \\
$\mathrm{C}(8)$ & $20(1)$ & $19(1)$ & $14(1)$ & $2(1)$ & $5(1)$ & $-1(1)$ \\
$\mathrm{C}(23)$ & $24(1)$ & $35(1)$ & $20(1)$ & $9(1)$ & $4(1)$ & $-1(1)$ \\
$\mathrm{C}(15)$ & $20(1)$ & $18(1)$ & $21(1)$ & $0(1)$ & $2(1)$ & $2(1)$ \\
$\mathrm{C}(14)$ & $20(1)$ & $16(1)$ & $16(1)$ & $-2(1)$ & $2(1)$ & $-3(1)$ \\
$\mathrm{C}(7)$ & $18(1)$ & $15(1)$ & $19(1)$ & $-4(1)$ & $2(1)$ & $-4(1)$ \\
$\mathrm{C}(11)$ & $18(1)$ & $32(1)$ & $22(1)$ & $6(1)$ & $6(1)$ & $4(1)$ \\
$\mathrm{C}(10)$ & $25(1)$ & $23(1)$ & $26(1)$ & $-4(1)$ & $4(1)$ & $3(1)$ \\
$\mathrm{C}(16)$ & $24(1)$ & $18(1)$ & $17(1)$ & $3(1)$ & $1(1)$ & $-2(1)$ \\
$\mathrm{C}(12)$ & $19(1)$ & $35(1)$ & $24(1)$ & $4(1)$ & $0(1)$ & $-7(1)$ \\
$\mathrm{C}(9)$ & $20(1)$ & $22(1)$ & $24(1)$ & $-5(1)$ & $0(1)$ & $-1(1)$ \\
$\mathrm{C}(13)$ & $25(1)$ & $23(1)$ & $22(1)$ & $-2(1)$ & $2(1)$ & $-6(1)$ \\
$\mathrm{C}(22)$ & $28(1)$ & $38(1)$ & $23(1)$ & $6(1)$ & $9(1)$ & $2(1)$ \\
$\mathrm{C}(2)$ & $20(1)$ & $18(1)$ & $18(1)$ & $1(1)$ & $4(1)$ & $-1(1)$ \\
$\mathrm{C}(4)$ & $23(1)$ & $20(1)$ & $18(1)$ & $-2(1)$ & $7(1)$ & $-4(1)$ \\
$\mathrm{C}(1)$ & $19(1)$ & $16(1)$ & $16(1)$ & $-3(1)$ & $5(1)$ & $-4(1)$ \\
$\mathrm{C}(20)$ & $29(1)$ & $31(1)$ & $22(1)$ & $7(1)$ & $4(1)$ & $5(1)$ \\
$\mathrm{C}(5)$ & $20(1)$ & $18(1)$ & $20(1)$ & $-2(1)$ & $5(1)$ & $-1(1)$ \\
$\mathrm{C}(3)$ & $24(1)$ & $16(1)$ & $16(1)$ & $-1(1)$ & $2(1)$ & $-5(1)$ \\
$\mathrm{C}(6)$ & $22(1)$ & $15(1)$ & $16(1)$ & $-2(1)$ & $4(1)$ & $-3(1)$ \\
$\mathrm{C}(21)$ & $24(1)$ & $25(1)$ & $16(1)$ & $4(1)$ & $5(1)$ & $-3(1)$ \\
$\mathrm{C}(1)$ & $22(1)$ & $17(1)$ & $21(1)$ & $-1(1)$ & $2(1)$ & $2(1)$ \\
$\mathrm{N}(2)$ & $24(1)$ & $28(1)$ & $19(1)$ & $7(1)$ & $5(1)$ & $1(1)$ \\
$\mathrm{N}(1)$ & $23(1)$ & $22(1)$ & $16(1)$ & $1(1)$ & $6(1)$ & $-2(1)$ \\
$\mathrm{Se}(1)$ & $20(1)$ & $20(1)$ & $14(1)$ & $3(1)$ & $6(1)$ & $3(1)$ \\
& & & & & &
\end{tabular}


Table 7S. Bond lengths $[\AA]$ and angles $\left[^{\circ}\right]$ for TMR-Se.

\begin{tabular}{|c|c|}
\hline $\mathrm{C}(18)-\mathrm{C}(19)$ & $1.386(2)$ \\
\hline $\mathrm{C}(18)-\mathrm{C}(17)$ & $1.407(2)$ \\
\hline C(18)-H(18) & 0.9500 \\
\hline$C(19)-C(14)$ & $1.424(2)$ \\
\hline $\mathrm{C}(19)-\mathrm{Se}(1)$ & $1.8779(17)$ \\
\hline $\mathrm{C}(17)-\mathrm{N}(2)$ & $1.358(2)$ \\
\hline$C(17)-C(16)$ & $1.424(3)$ \\
\hline $\mathrm{C}(8)-\mathrm{C}(9)$ & $1.393(3)$ \\
\hline C(8)-C(13) & $1.394(3)$ \\
\hline $\mathrm{C}(8)-\mathrm{C}(7)$ & $1.500(2)$ \\
\hline $\mathrm{C}(23)-\mathrm{N}(2)$ & $1.453(2)$ \\
\hline $\mathrm{C}(23)-\mathrm{H}(23 \mathrm{~A})$ & 0.9800 \\
\hline $\mathrm{C}(23)-\mathrm{H}(23 \mathrm{~B})$ & 0.9800 \\
\hline $\mathrm{C}(23)-\mathrm{H}(23 \mathrm{C})$ & 0.9800 \\
\hline$C(15)-C(16)$ & $1.364(2)$ \\
\hline$C(15)-C(14)$ & $1.423(2)$ \\
\hline $\mathrm{C}(15)-\mathrm{H}(15)$ & 0.9500 \\
\hline $\mathrm{C}(14)-\mathrm{C}(7)$ & $1.434(2)$ \\
\hline$C(7)-C(6)$ & $1.395(2)$ \\
\hline $\mathrm{C}(11)-\mathrm{C}(12)$ & $1.381(3)$ \\
\hline $\mathrm{C}(11)-\mathrm{C}(10)$ & $1.383(3)$ \\
\hline $\mathrm{C}(11)-\mathrm{H}(11)$ & 0.9500 \\
\hline $\mathrm{C}(10)-\mathrm{C}(9)$ & $1.386(3)$ \\
\hline $\mathrm{C}(10)-\mathrm{H}(10)$ & 0.9500 \\
\hline $\mathrm{C}(16)-\mathrm{H}(16)$ & 0.9500 \\
\hline $\mathrm{C}(12)-\mathrm{C}(13)$ & $1.389(3)$ \\
\hline $\mathrm{C}(12)-\mathrm{H}(12)$ & 0.9500 \\
\hline $\mathrm{C}(9)-\mathrm{H}(9)$ & 0.9500 \\
\hline $\mathrm{C}(13)-\mathrm{H}(13)$ & 0.9500 \\
\hline$C(22)-N(2)$ & $1.468(3)$ \\
\hline $\mathrm{C}(22)-\mathrm{H}(22 \mathrm{~A})$ & 0.9800 \\
\hline $\mathrm{C}(22)-\mathrm{H}(22 \mathrm{~B})$ & 0.9800 \\
\hline $\mathrm{C}(22)-\mathrm{H}(22 \mathrm{C})$ & 0.9800 \\
\hline$C(2)-C(1)$ & $1.371(3)$ \\
\hline$C(2)-C(3)$ & $1.419(2)$ \\
\hline $\mathrm{C}(2)-\mathrm{H}(2)$ & 0.9500 \\
\hline$C(4)-C(5)$ & $1.357(3)$ \\
\hline$C(4)-C(3)$ & $1.439(3)$ \\
\hline $\mathrm{C}(4)-\mathrm{H}(4)$ & 0.9500 \\
\hline C(1)-C(6) & $1.438(3)$ \\
\hline C(1)-Se(1) & $1.8798(17)$ \\
\hline $\mathrm{C}(20)-\mathrm{N}(1)$ & $1.482(2)$ \\
\hline $\mathrm{C}(20)-\mathrm{H}(20 \mathrm{~A})$ & 0.9800 \\
\hline $\mathrm{C}(20)-\mathrm{H}(20 \mathrm{~B})$ & 0.9800 \\
\hline $\mathrm{C}(20)-\mathrm{H}(20 \mathrm{C})$ & 0.9800 \\
\hline$C(5)-C(6)$ & $1.444(2)$ \\
\hline $\mathrm{C}(5)-\mathrm{H}(5)$ & 0.9500 \\
\hline C(3)-N(1) & $1.336(2)$ \\
\hline $\mathrm{C}(21)-\mathrm{N}(1)$ & $1.463(2)$ \\
\hline $\mathrm{C}(21)-\mathrm{H}(21 \mathrm{~A})$ & 0.9800 \\
\hline $\mathrm{C}(21)-\mathrm{H}(21 \mathrm{~B})$ & 0.9800 \\
\hline $\mathrm{C}(21)-\mathrm{H}(21 \mathrm{C})$ & 0.9800 \\
\hline $\mathrm{C}(19)-\mathrm{C}(18)-\mathrm{C}(17)$ & $121.14(17)$ \\
\hline
\end{tabular}




\begin{tabular}{|c|c|}
\hline $\mathrm{C}(19)-\mathrm{C}(18)-\mathrm{H}(18)$ & 119.4 \\
\hline $\mathrm{C}(17)-\mathrm{C}(18)-\mathrm{H}(18)$ & 119.4 \\
\hline$C(18)-C(19)-C(14)$ & $122.27(16)$ \\
\hline$C(18)-C(19)-\operatorname{Se}(1)$ & $115.14(13)$ \\
\hline $\mathrm{C}(14)-\mathrm{C}(19)-\mathrm{Se}(1)$ & $122.58(13)$ \\
\hline N(2)-C(17)-C(18) & $121.07(17)$ \\
\hline $\mathrm{N}(2)-\mathrm{C}(17)-\mathrm{C}(16)$ & $121.53(16)$ \\
\hline$C(18)-C(17)-C(16)$ & $117.40(16)$ \\
\hline $\mathrm{C}(9)-\mathrm{C}(8)-\mathrm{C}(13)$ & $119.03(17)$ \\
\hline C(9)-C(8)-C(7) & $120.16(16)$ \\
\hline $\mathrm{C}(13)-\mathrm{C}(8)-\mathrm{C}(7)$ & $120.78(16)$ \\
\hline$C(16)-C(15)-C(14)$ & $123.44(17)$ \\
\hline $\mathrm{C}(16)-\mathrm{C}(15)-\mathrm{H}(15)$ & 118.3 \\
\hline $\mathrm{C}(14)-\mathrm{C}(15)-\mathrm{H}(15)$ & 118.3 \\
\hline$C(15)-C(14)-C(19)$ & $115.03(15)$ \\
\hline $\mathrm{C}(15)-\mathrm{C}(14)-\mathrm{C}(7)$ & $120.30(16)$ \\
\hline $\mathrm{C}(19)-\mathrm{C}(14)-\mathrm{C}(7)$ & $124.66(16)$ \\
\hline $\mathrm{C}(6)-\mathrm{C}(7)-\mathrm{C}(14)$ & $125.41(17)$ \\
\hline $\mathrm{C}(6)-\mathrm{C}(7)-\mathrm{C}(8)$ & $118.47(15)$ \\
\hline C(14)-C(7)-C(8) & $116.12(15)$ \\
\hline $\mathrm{C}(12)-\mathrm{C}(11)-\mathrm{C}(10)$ & $119.74(18)$ \\
\hline $\mathrm{C}(12)-\mathrm{C}(11)-\mathrm{H}(11)$ & 120.1 \\
\hline $\mathrm{C}(10)-\mathrm{C}(11)-\mathrm{H}(11)$ & 120.1 \\
\hline $\mathrm{C}(11)-\mathrm{C}(10)-\mathrm{C}(9)$ & $119.90(18)$ \\
\hline $\mathrm{C}(11)-\mathrm{C}(10)-\mathrm{H}(10)$ & 120.1 \\
\hline $\mathrm{C}(9)-\mathrm{C}(10)-\mathrm{H}(10)$ & 120.1 \\
\hline$C(15)-C(16)-C(17)$ & $120.67(17)$ \\
\hline $\mathrm{C}(15)-\mathrm{C}(16)-\mathrm{H}(16)$ & 119.7 \\
\hline $\mathrm{C}(17)-\mathrm{C}(16)-\mathrm{H}(16)$ & 119.7 \\
\hline $\mathrm{C}(11)-\mathrm{C}(12)-\mathrm{C}(13)$ & $120.82(18)$ \\
\hline $\mathrm{C}(11)-\mathrm{C}(12)-\mathrm{H}(12)$ & 119.6 \\
\hline $\mathrm{C}(13)-\mathrm{C}(12)-\mathrm{H}(12)$ & 119.6 \\
\hline $\mathrm{C}(10)-\mathrm{C}(9)-\mathrm{C}(8)$ & $120.78(17)$ \\
\hline $\mathrm{C}(10)-\mathrm{C}(9)-\mathrm{H}(9)$ & 119.6 \\
\hline $\mathrm{C}(8)-\mathrm{C}(9)-\mathrm{H}(9)$ & 119.6 \\
\hline $\mathrm{C}(12)-\mathrm{C}(13)-\mathrm{C}(8)$ & $119.72(18)$ \\
\hline $\mathrm{C}(12)-\mathrm{C}(13)-\mathrm{H}(13)$ & 120.1 \\
\hline $\mathrm{C}(8)-\mathrm{C}(13)-\mathrm{H}(13)$ & 120.1 \\
\hline $\mathrm{C}(1)-\mathrm{C}(2)-\mathrm{C}(3)$ & $121.15(17)$ \\
\hline $\mathrm{C}(1)-\mathrm{C}(2)-\mathrm{H}(2)$ & 119.4 \\
\hline $\mathrm{C}(3)-\mathrm{C}(2)-\mathrm{H}(2)$ & 119.4 \\
\hline$C(5)-C(4)-C(3)$ & $120.40(17)$ \\
\hline $\mathrm{C}(5)-\mathrm{C}(4)-\mathrm{H}(4)$ & 119.8 \\
\hline $\mathrm{C}(3)-\mathrm{C}(4)-\mathrm{H}(4)$ & 119.8 \\
\hline $\mathrm{C}(2)-\mathrm{C}(1)-\mathrm{C}(6)$ & $122.52(16)$ \\
\hline$C(2)-C(1)-\operatorname{Se}(1)$ & $114.64(13)$ \\
\hline $\mathrm{C}(6)-\mathrm{C}(1)-\mathrm{Se}(1)$ & $122.81(13)$ \\
\hline$C(4)-C(5)-C(6)$ & $123.30(17)$ \\
\hline $\mathrm{C}(4)-\mathrm{C}(5)-\mathrm{H}(5)$ & 118.4 \\
\hline $\mathrm{C}(6)-\mathrm{C}(5)-\mathrm{H}(5)$ & 118.4 \\
\hline $\mathrm{N}(1)-\mathrm{C}(3)-\mathrm{C}(2)$ & $120.73(17)$ \\
\hline $\mathrm{N}(1)-\mathrm{C}(3)-\mathrm{C}(4)$ & $121.56(16)$ \\
\hline$C(2)-C(3)-C(4)$ & $117.70(16)$ \\
\hline$C(7)-C(6)-C(1)$ & $124.44(16)$ \\
\hline$C(7)-C(6)-C(5)$ & $120.71(16)$ \\
\hline$C(1)-C(6)-C(5)$ & $114.86(16)$ \\
\hline
\end{tabular}


$\mathrm{C}(17)-\mathrm{N}(2)-\mathrm{C}(23)$

$\mathrm{C}(17)-\mathrm{N}(2)-\mathrm{C}(22)$

$\mathrm{C}(23)-\mathrm{N}(2)-\mathrm{C}(22)$

$\mathrm{C}(3)-\mathrm{N}(1)-\mathrm{C}(21)$

$\mathrm{C}(3)-\mathrm{N}(1)-\mathrm{C}(20)$

$\mathrm{C}(21)-\mathrm{N}(1)-\mathrm{C}(20)$

$\mathrm{C}(19)-\mathrm{Se}(1)-\mathrm{C}(1)$
120.81(16)

120.59(16)

$118.35(15)$

121.37(16)

121.21(15)

117.41(15)

99.89(8) 


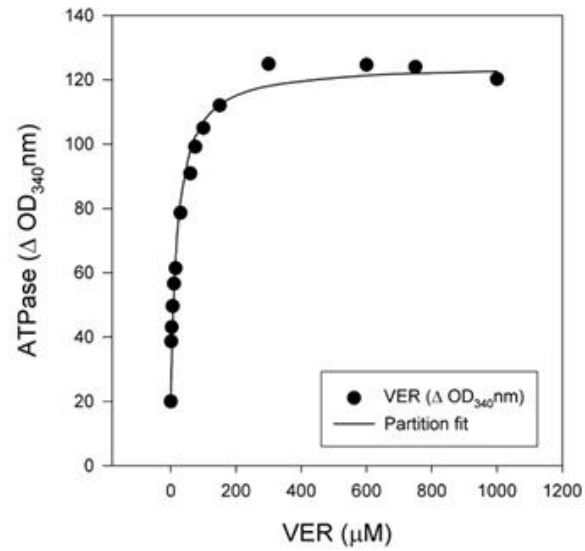

Verapamil

Figure 2S. Partition fit of ATPase activity for various concentrations of VER, TMR, R6G, R123, TMR-Se, 1-12, 14-17, and 20-22. 

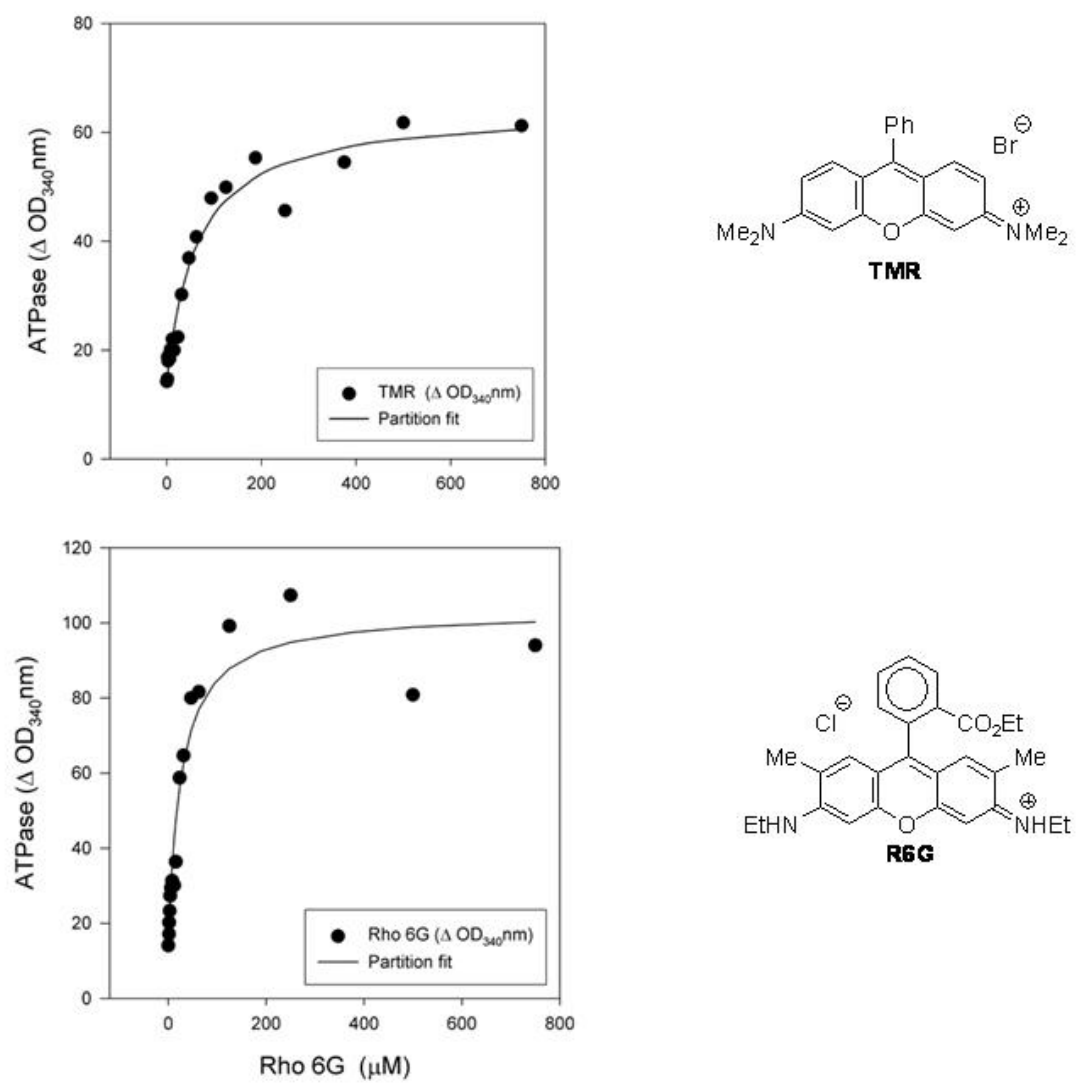

Figure 2S. - contd- 

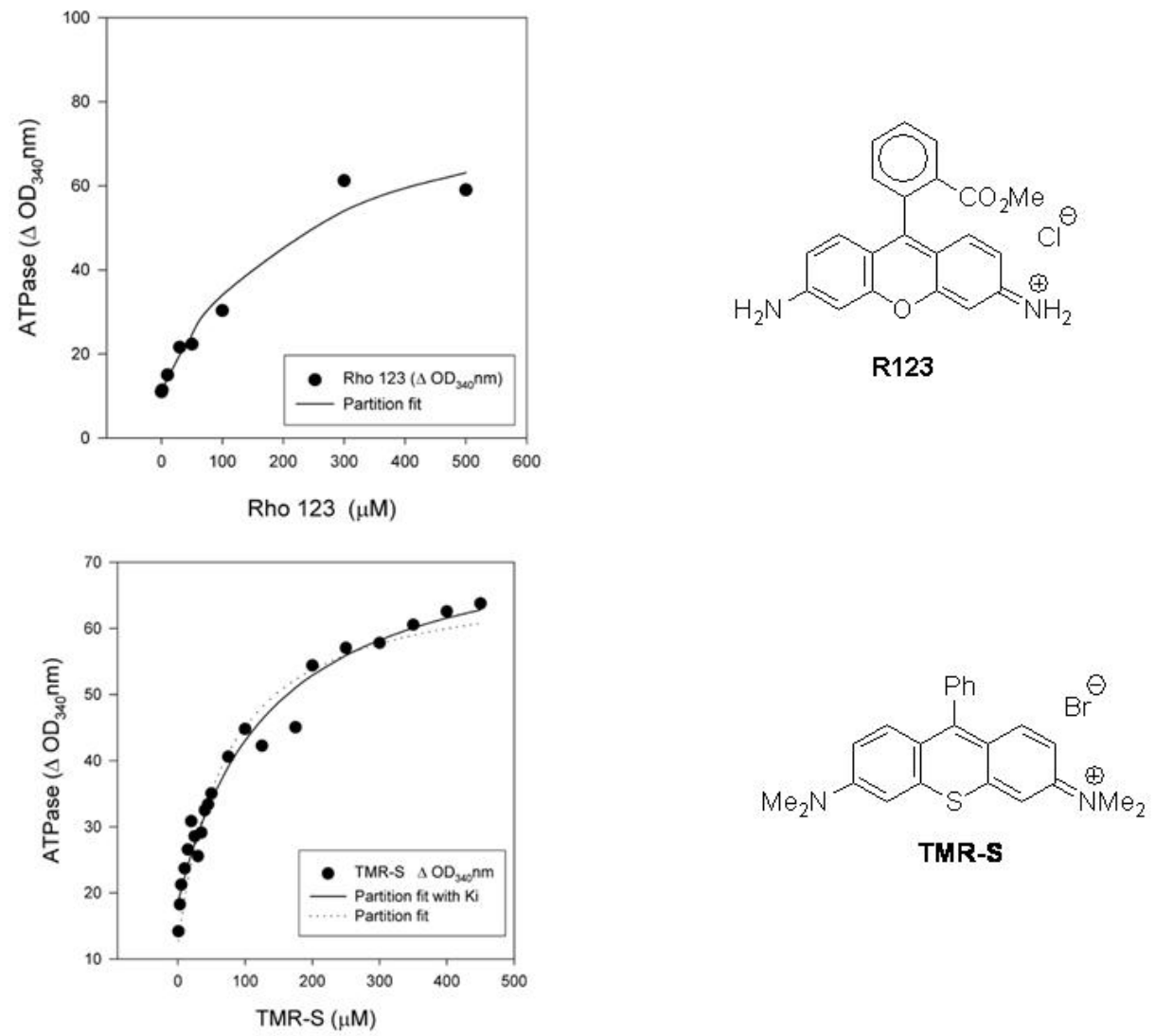

Figure 2S. - contd- 

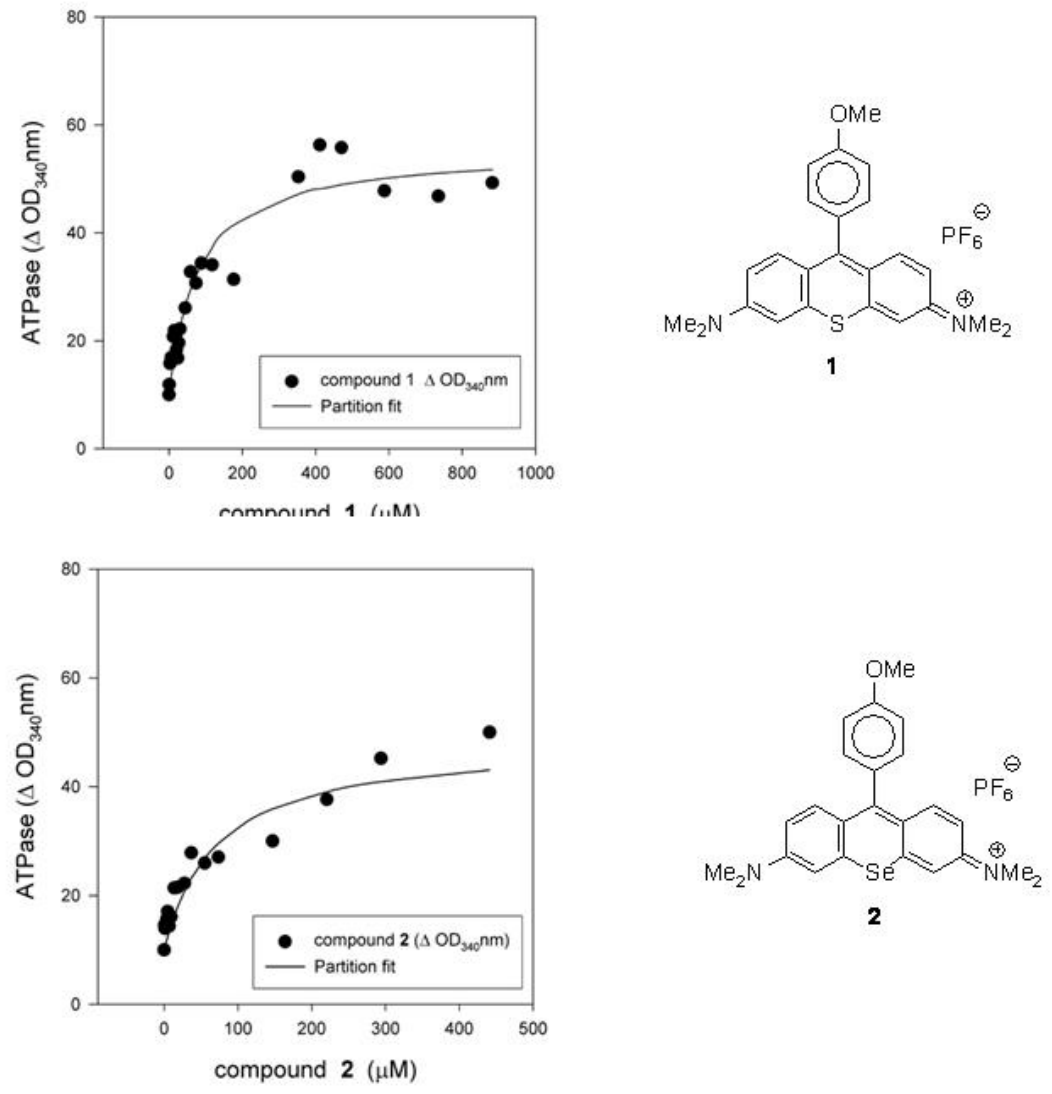

Figure 2S. - contd- 

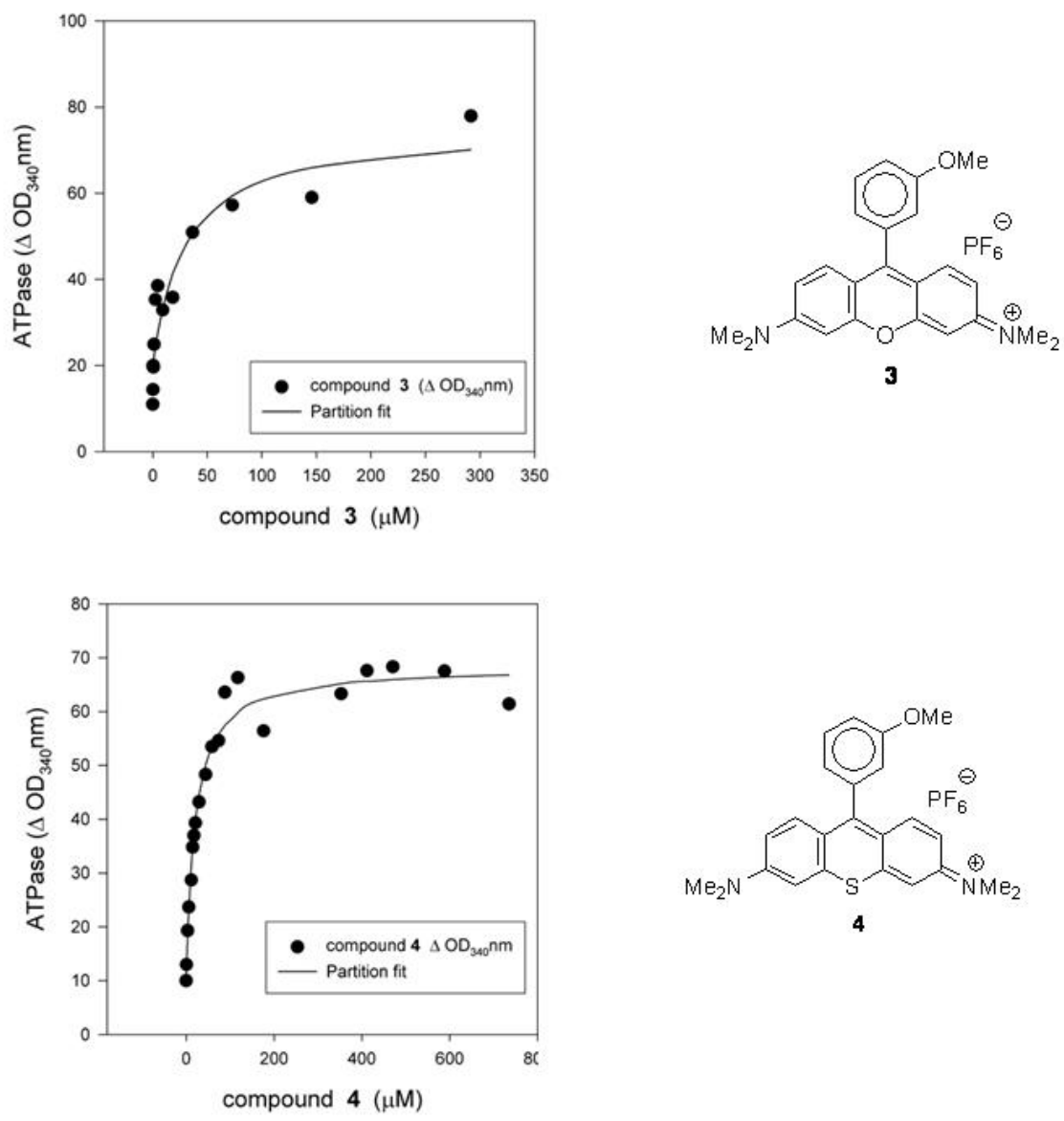

Figure 2S. - contd- 

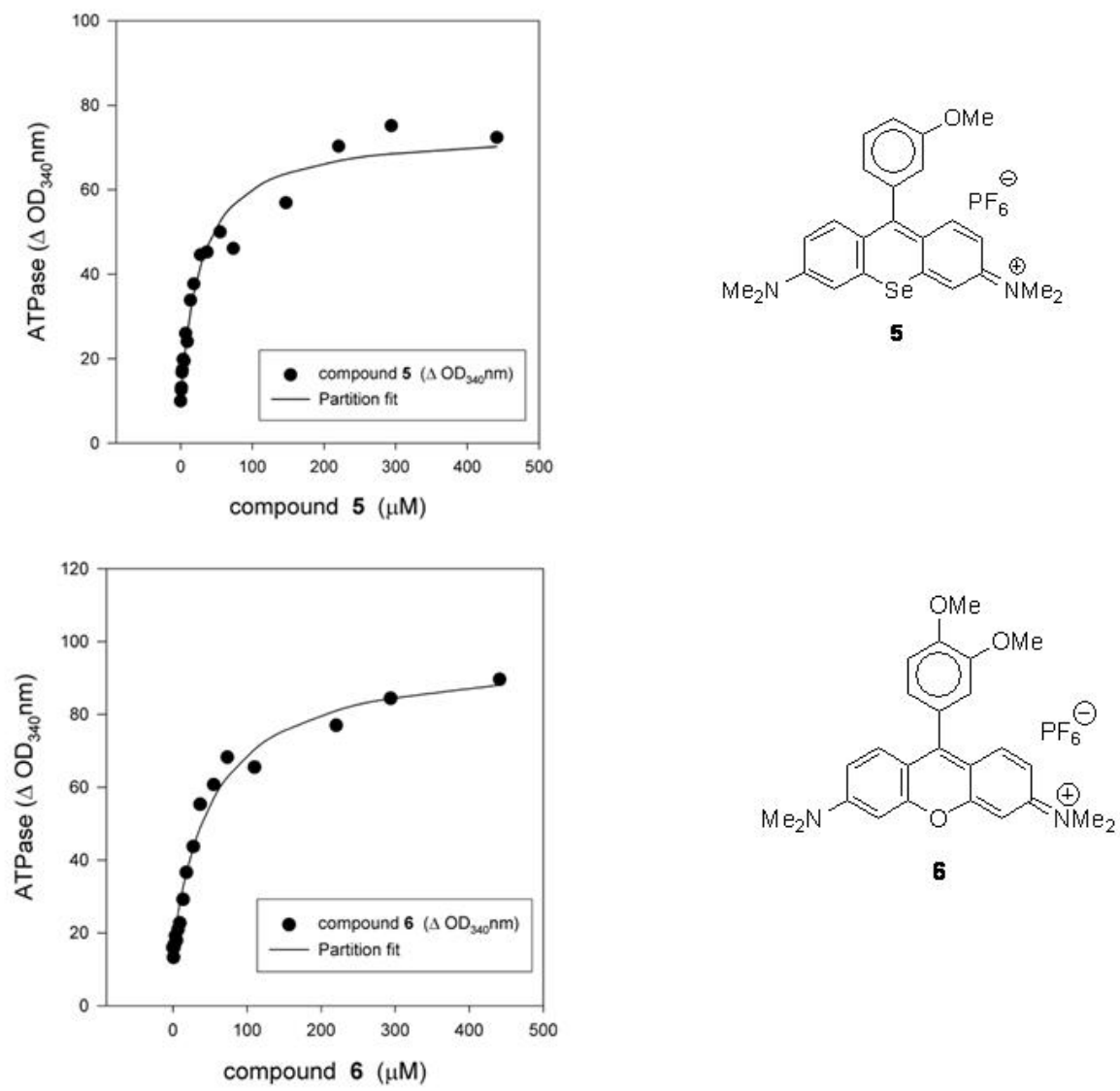

Figure 2S. - contd- 

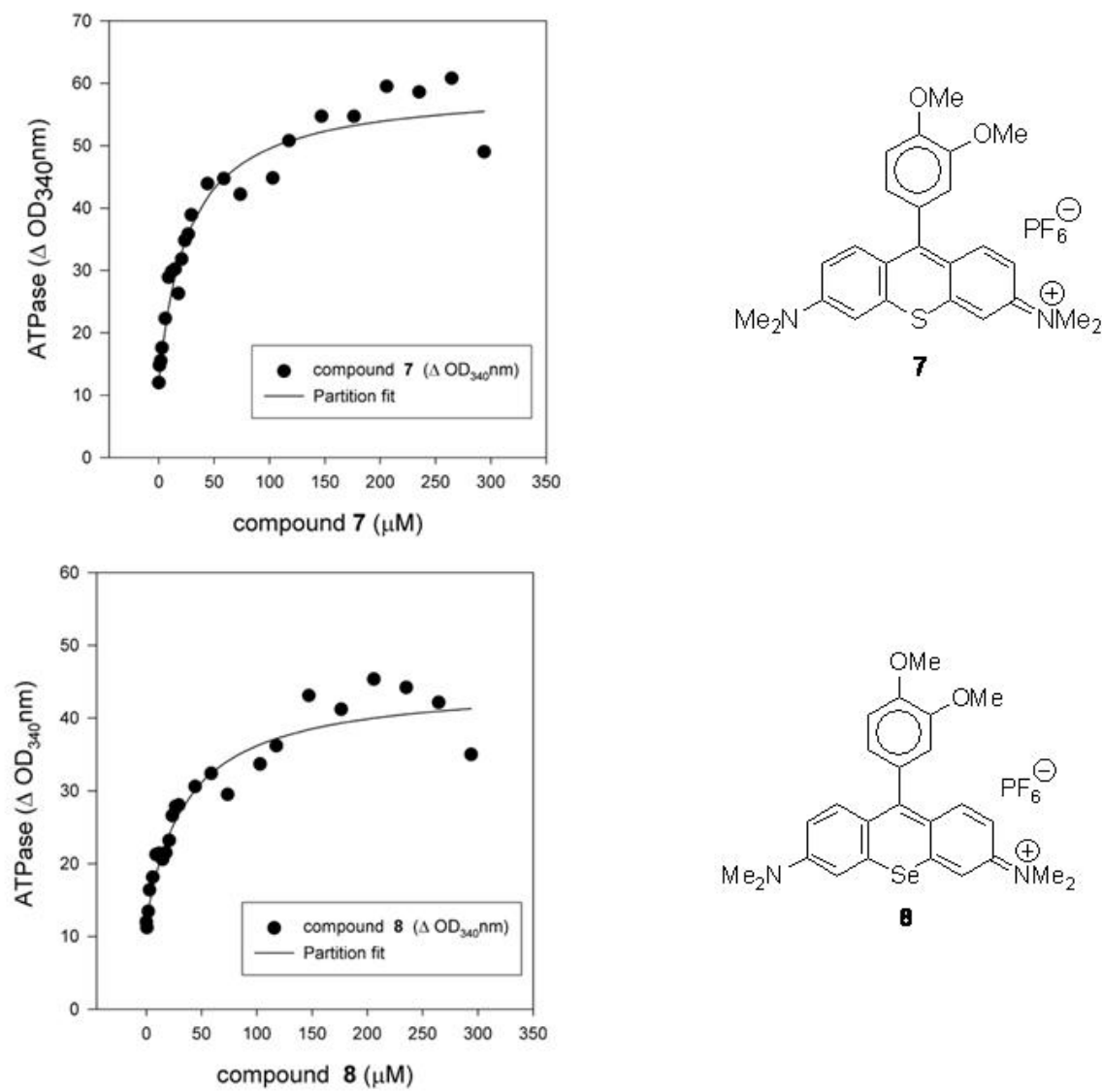

Figure 2S. - contd- 

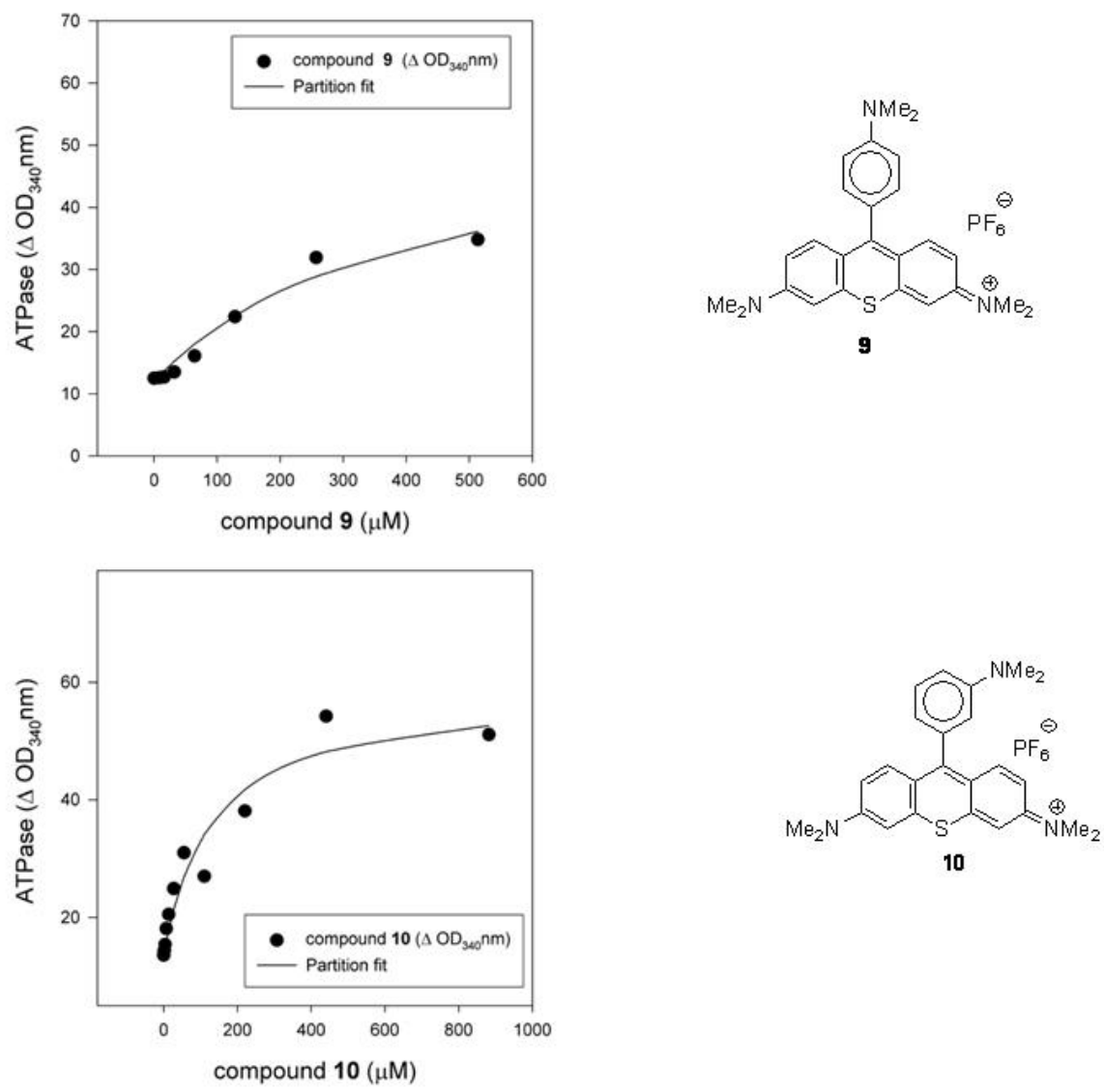

Figure 2S. - contd- 

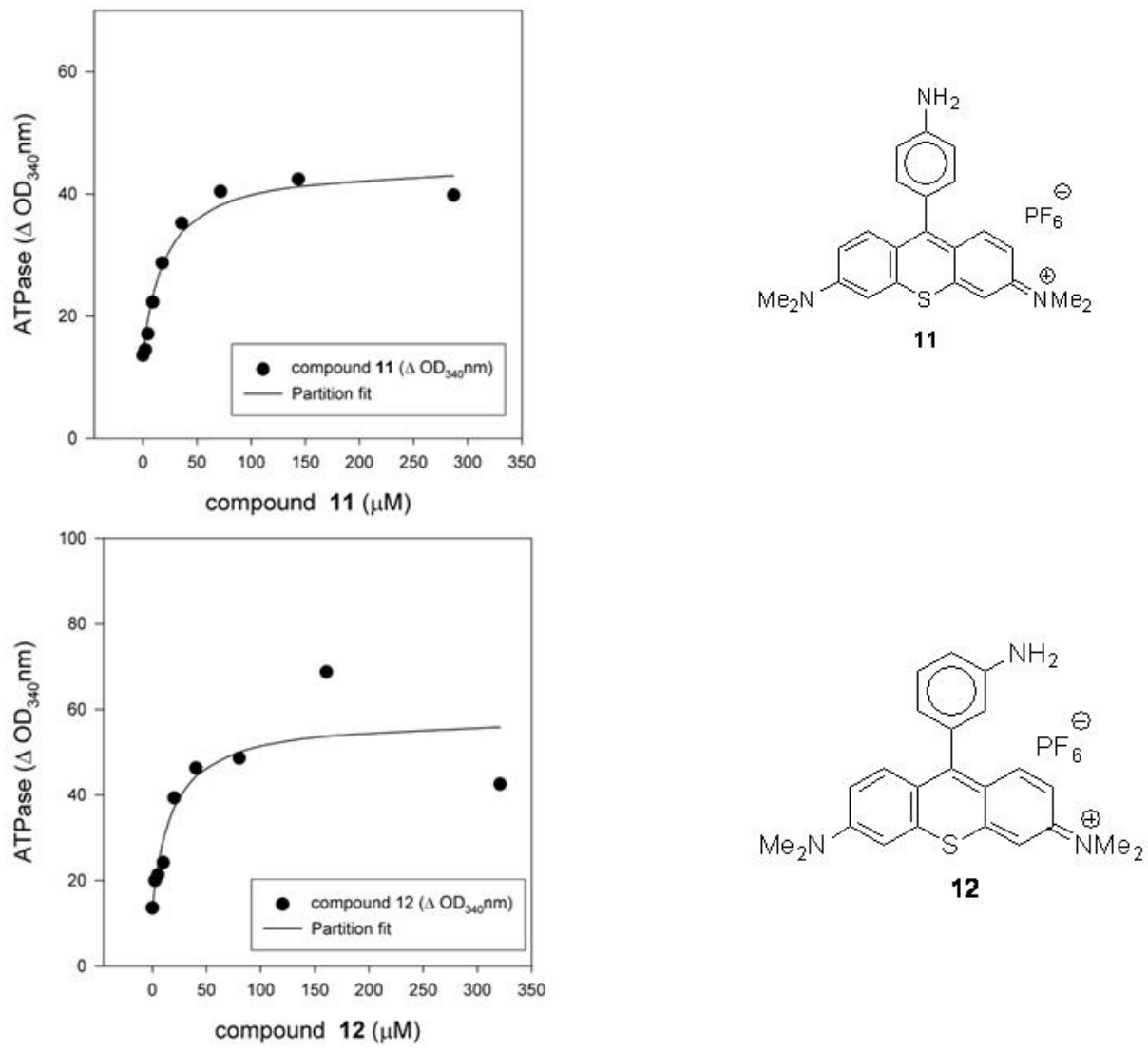

Figure 2S. - contd- 

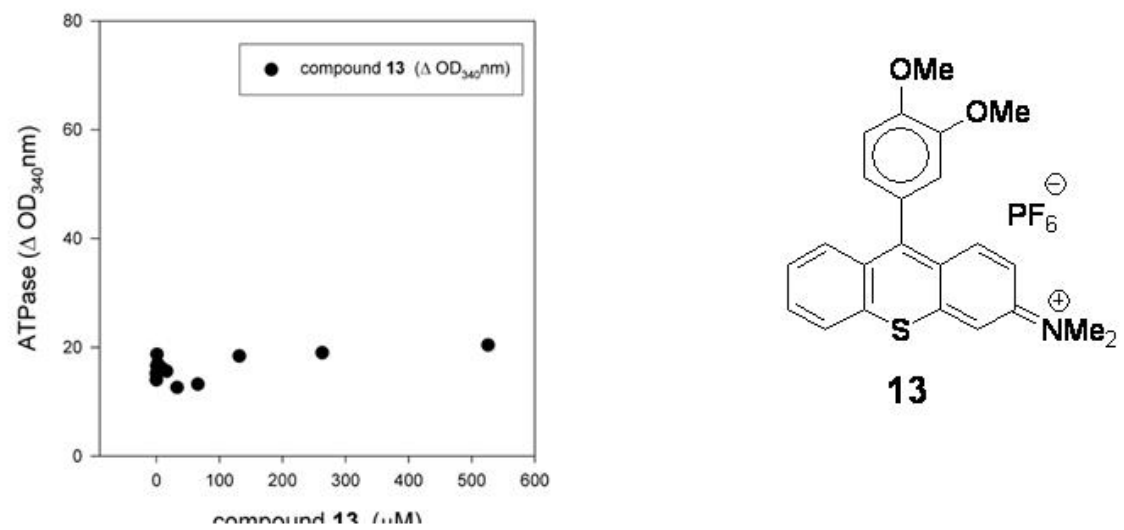

13
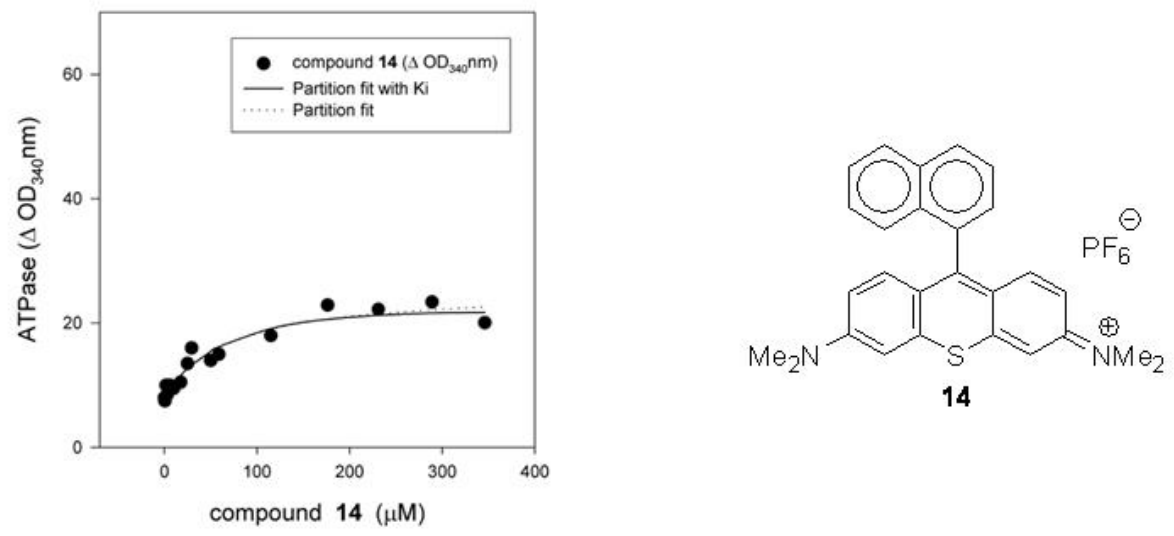

Figure 2S. - contd- 

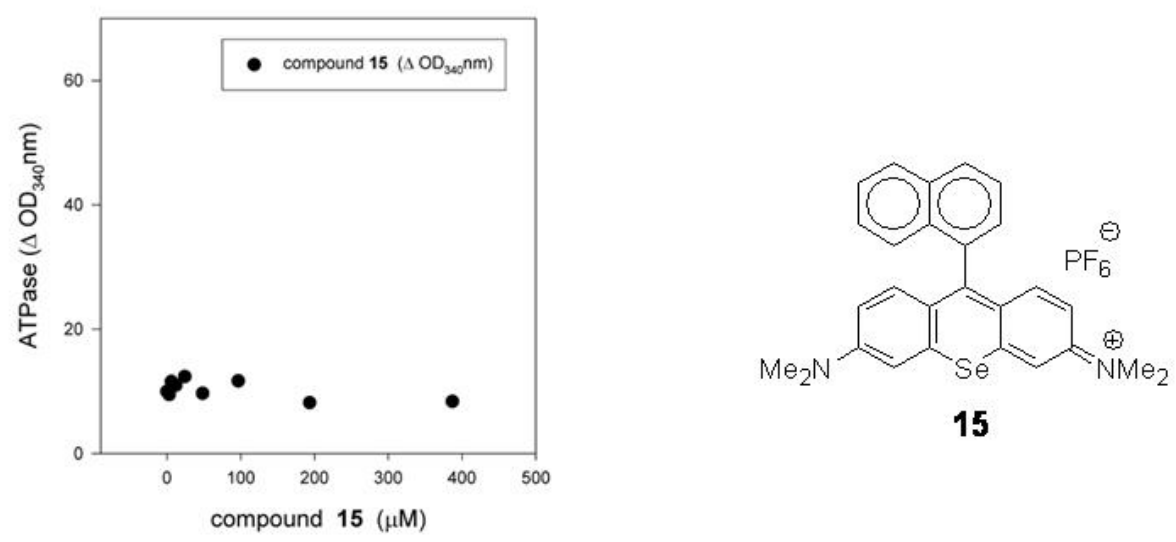

15
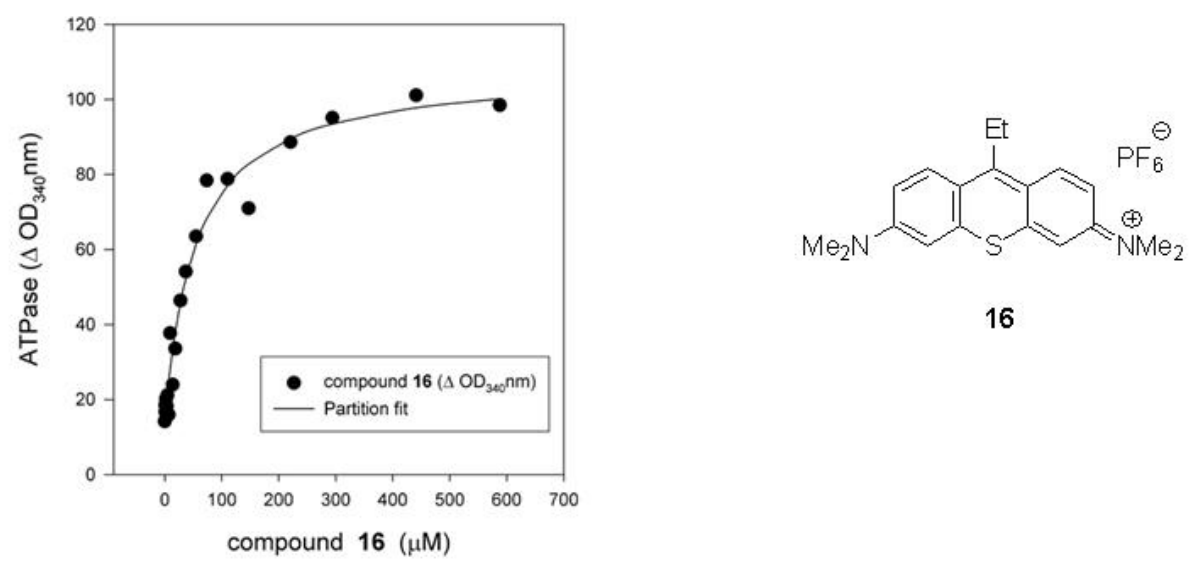

16

Figure 2S. - contd- 

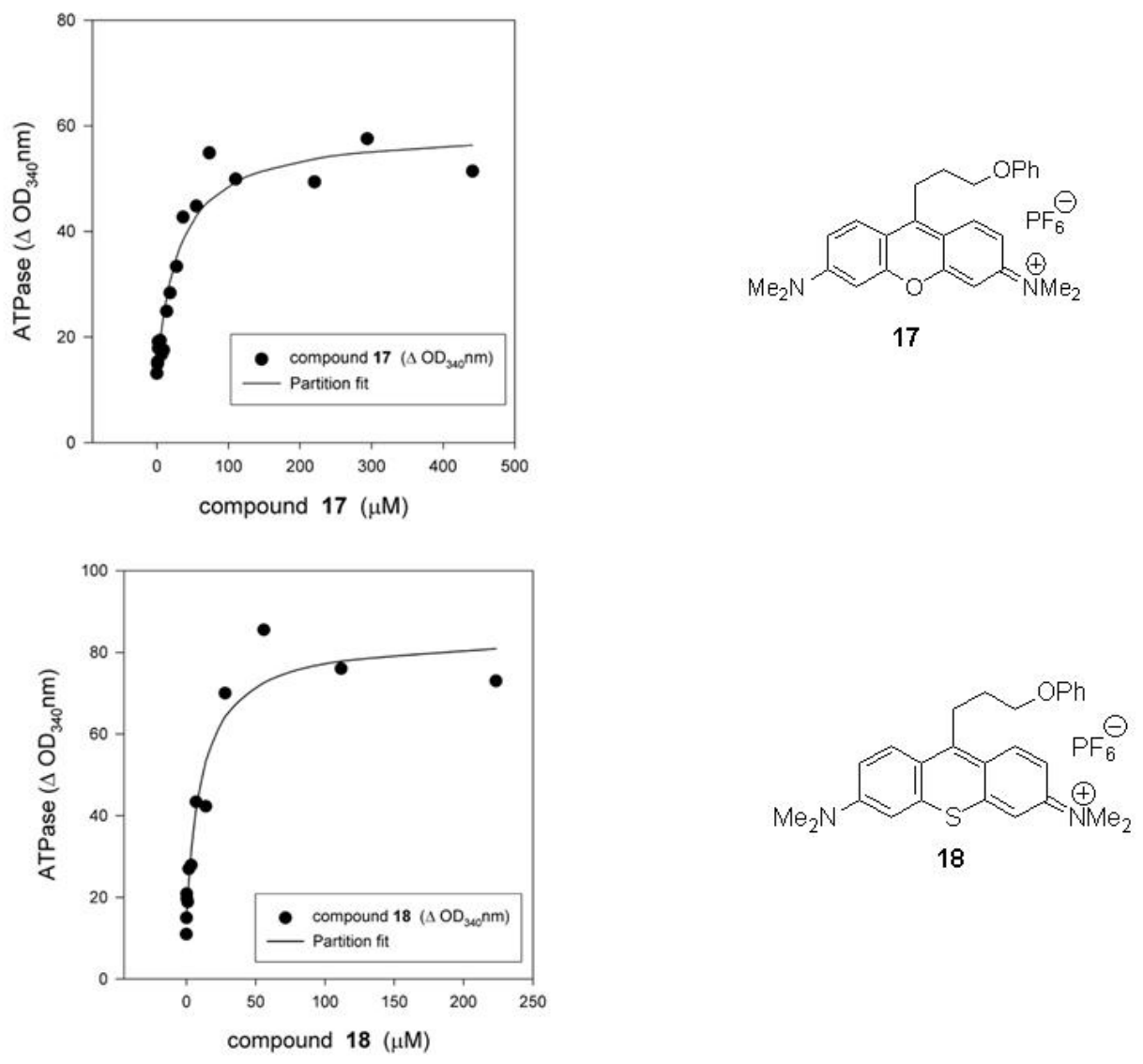

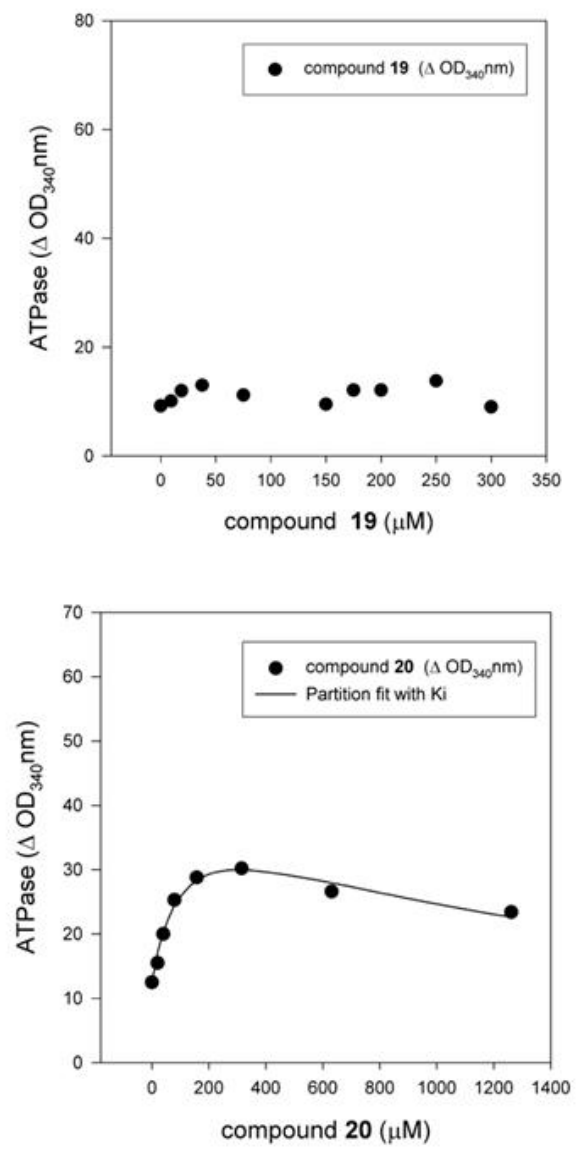
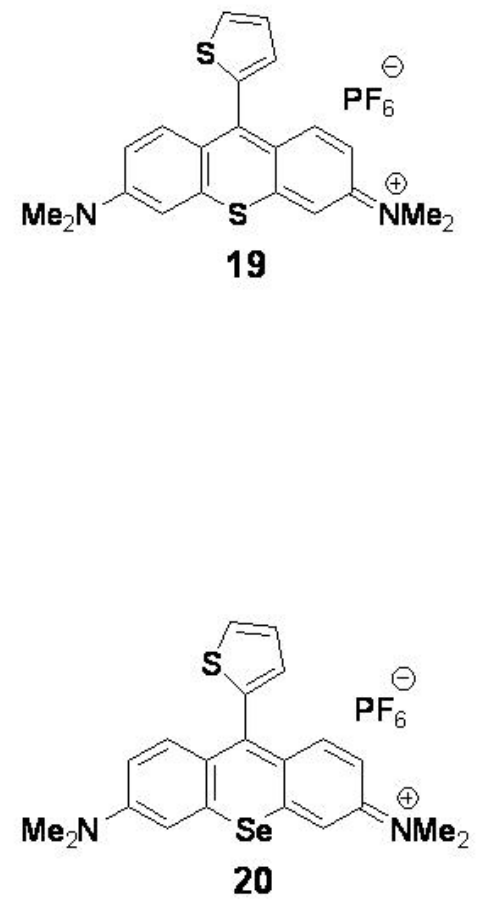

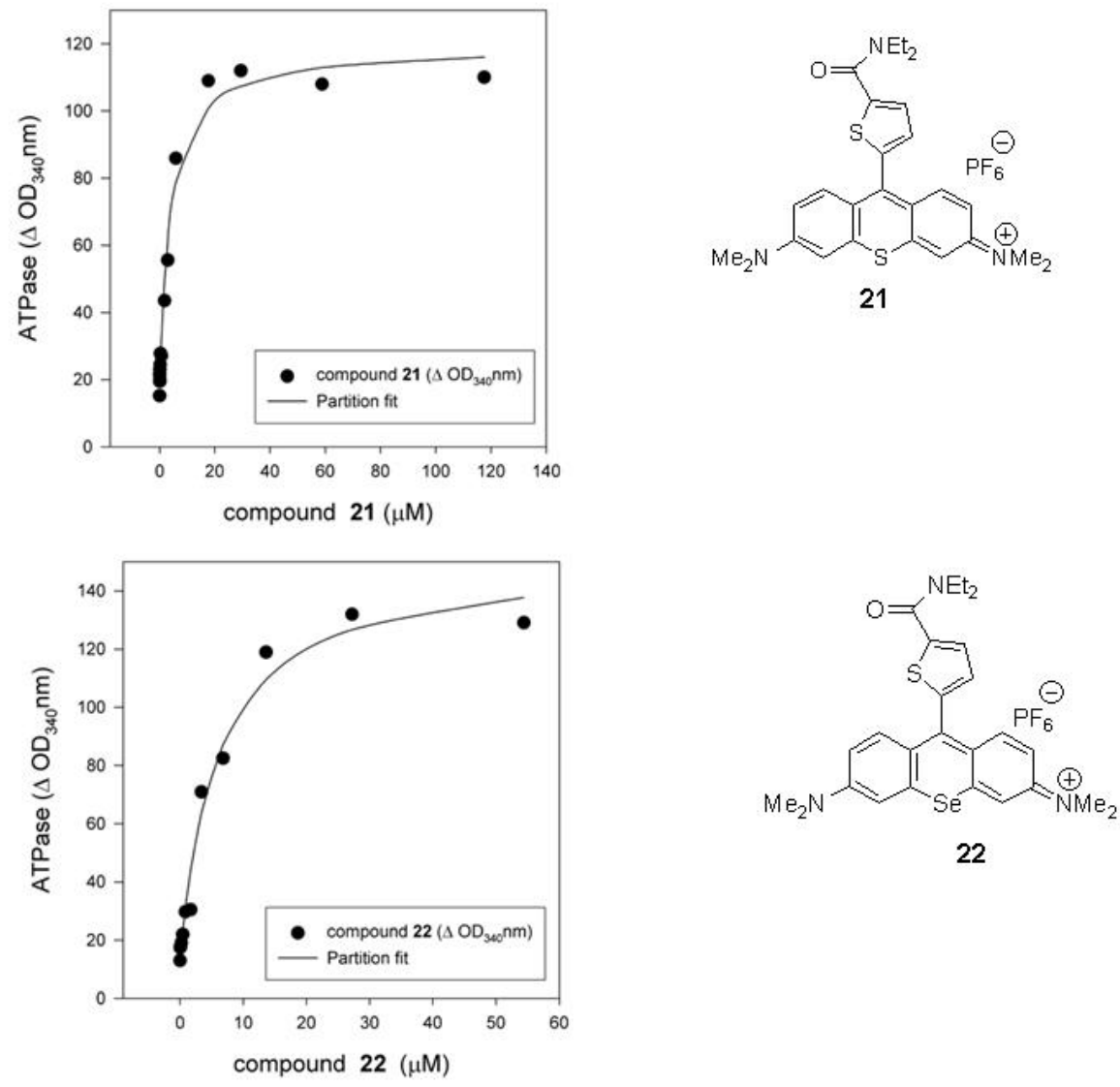

Figure 2S. -contd- 


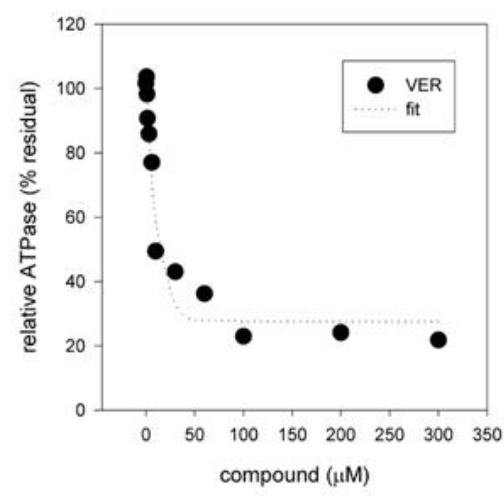

b) ADP-Vi trapping by wild-type MDR3 with Verapamil

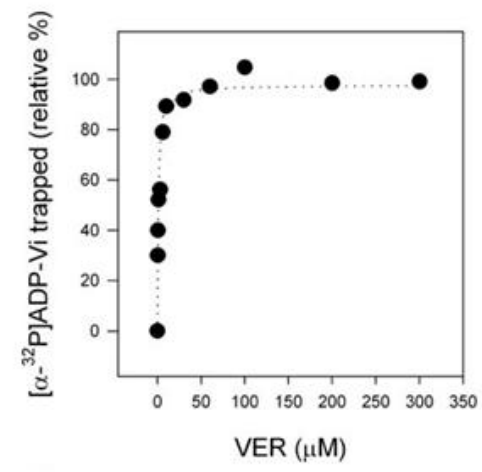

a) $\mathrm{ADP}$-Vi trapping from the reverse direction of the normal catalytic pathway by lipidactivated wild-type Pgp with Verapamil c) Tight ATP binding by $E_{552} A / E_{1197} A$ (A/A) mutant MDR3 with Verapamil

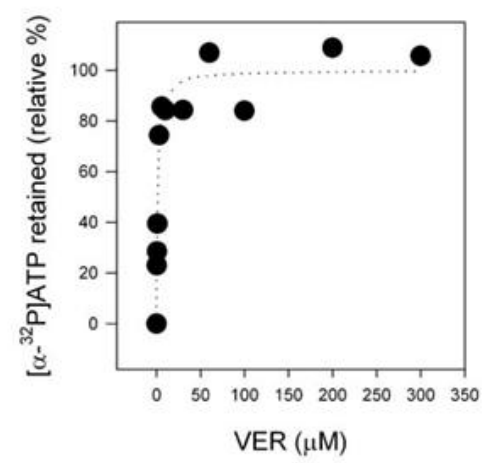

Figure 3S. Effect of Verapamil on ATP catalytic site. 


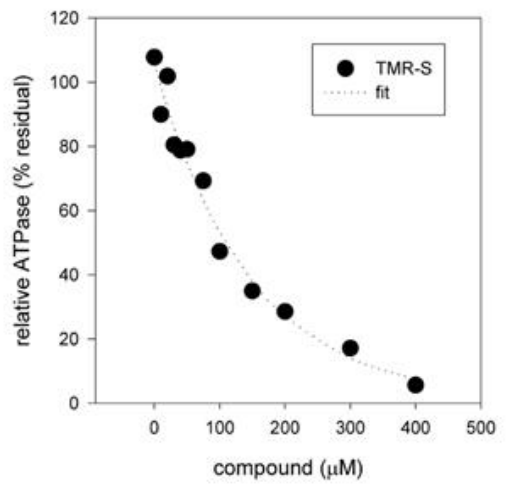

a) ADP-Vi trapping from the reverse direction of the normal catalytic pathway by lipidactivated wild-type Pgp with TMR-S

b) ADP-Vi trapping by wild-type MDR3 with TMR-S

c) Tight ATP binding by $E_{552} A / E_{1197} A$ (A/A) mutant MDR3 with TMR-S
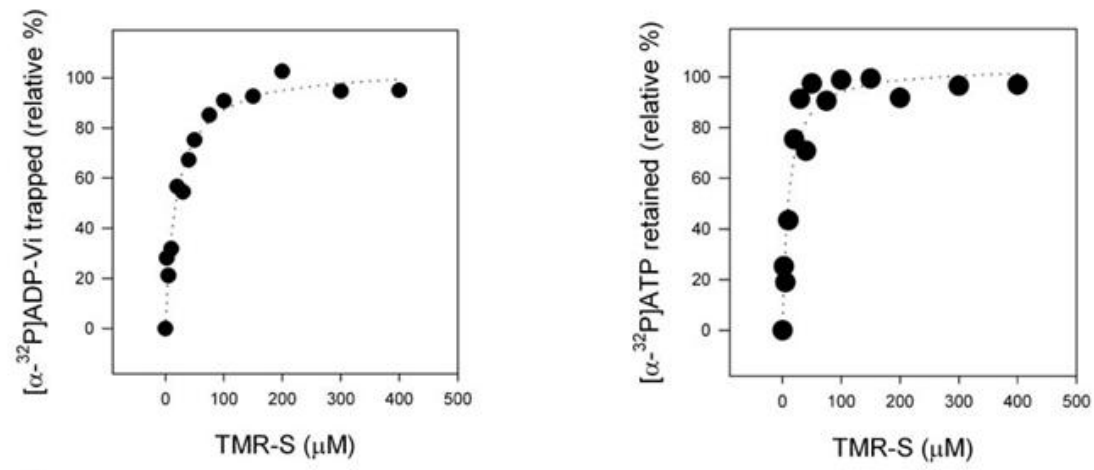

Figure 4S. Effect of TMR-S on ATP catalytic site. 